\title{
Estimates from atomic models of tension-shear coupling in dislocation nucleation from a crack tip
}

\author{
Yuemin Sun, Glenn E. Beltz* and James R. Rice** \\ Division of Applied Sciences, Harvard University, Cambridge, MA 02138 (USA)
}

\begin{abstract}
The normal stress distribution across a slip plane has the effect of reducing the critical loading required for dislocation emission from a crack tip. The reduction by normal stresses was found to be very significant for $\mathrm{Si}$, based on properties estimated for it using density functional theory, to be large for Fe as modeled by the embedded atom method (EAM), and to be smaller in $\mathrm{Al}, \mathrm{Ni}$ and ordered $\mathrm{Ni}_{3} \mathrm{Al}$, estimated using the EAM. The general dependence over a wide range on parameters characterizing the tension-shear coupling was also determined. In the context of a Peierls model for dislocation nucleation at a crack tip (J. R. Rice, J. Mech. Phys. Solids, 40 (1992) 239), our approach was to search for onset of the dislocation nucleation instability based on the numerical solution of the system of non-linear integral equations describing an incipient dislocation. The incipient dislocation consists of a distribution of sliding and opening displacements along a slip plane emanating from the crack tip; these displacements are related to the shear and tensile stresses across the slip plane by constitutive relations based on the atomic models mentioned. Results from the atomic models are used to parametrize constitutive relations involving a Frenkel sinusoidal dependence of shear stress on sliding displacement at any fixed opening displacement, and a Rose-Ferrante-Smith universal binding form of dependence of tensile stress on opening displacement at any fixed shear displacement. These relations then enter the system of integral equations, solved numerically, which describe the elasticity solution for a non-uniform distribution of sliding and opening along the slip plane. The results show that tension-shear coupling will often significantly reduce the loading for dislocation emission from the value estimated on the basis of an unstable stacking energy $\gamma_{u s}$ determined with neglect of such coupling, in a shear-only type analysis. For the EAM models of the metals considered, a simple and approximate method to account for the tension effects is to use a modified quantity $\gamma_{\mathrm{us}}{ }^{\left({ }^{*}\right)}$, which is an unstable stacking energy for lattice planes which are constrained to a fixed opening $\Delta_{\theta}{ }^{*}$, corresponding to that for vanishing normal stress at the unstable shear equilibrium position. Moreover, it is found that the normal stress effect can be described well in these cases by replacing the unstable stacking energy $\gamma_{u s}$ in the shear-only model by a tension softened $\gamma_{u s}(\psi)$, which depends on the phase angle $\psi$ of the combined tension-shear loading along the slip plane according to the stress intensity factors of the elastic singular solution. The same simple procedures for accounting for tension effects on nucleation are less suitable for lattices with strong coupling such as $\mathrm{Si}$.
\end{abstract}

\section{Introduction}

A new analysis of dislocation emission from a crack tip has been given by Rice [1], based on the Peierls [2] concept of a periodic relation between shear stress and sliding displacement along a slip plane embedded in an elastic continuum. This combines continuum elasticity with atomistic descriptions of the dislocation core in a simple way. Rice applied that concept along a slip plane emanating from a stressed crack tip and, in an analysis that considered shear only (i.e. without coupling of the sliding displacements to tensile stress

\footnotetext{
* Present affiliation: Department of Mechanical and Environmental Engineering, University of California, Santa Barbara, CA 93106, USA. Address until 1/1/94: Max-Planck-Institut für Metallforschung, Institut für Werkstoffwissenschaft, Seestraße 92, D-70174 Stuttgart, Germany.

**Also at the Department of Earth and Planetary Sciences, Harvard University, Cambridge, MA 02138, USA.
}

across the slip plane), derived an exact solution for the dislocation nucleation condition when the slip plane and crack plane coincide (corresponding to $\theta=0$ in Fig. 1). His analysis introduced a new solid state parameter $\gamma_{u s}$, termed the unstable stacking energy; it is defined as the energy per unit area of slip plane when the lattice on one side of the plane is shifted in shear, relative to the lattice on the other side, to the unstable equilibrium position at or near to a sliding displacement of $b / 2$, where $b$ is the Burgers vector to form a complete dislocation. That is, $\gamma_{u s}$ is the height of the energy saddle point traversed in going from the minimum energy state at zero sliding to that at the sliding $b$; here, in application to different materials, $b$ may correspond to a full dislocation, or to a Shockley partial in an f.c.c. lattice, or to a constituent dislocation of a superlattice dislocation in an ordered alloy. Indeed, $\gamma_{u s}$ is an energy of the same type, but larger, than the stacking fault energy $\gamma_{\mathrm{sf}}$, or antiphase boundary energy $\gamma_{\mathrm{APB}}$, entering these latter types of defects. 


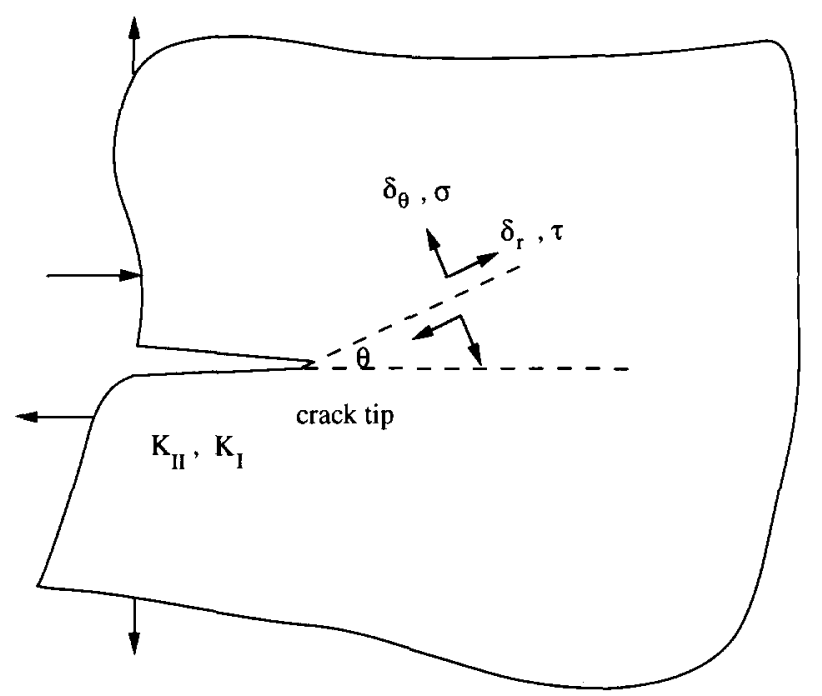

Fig. 1. An incipient dislocation, represented by a distribution of sliding and opening displacement, develops along a slip plane, tilted at angle $\theta$ with respect to the crack plane, in response to mixed loading $K_{\mathrm{II}}$ and $K_{\mathrm{l}}$.

Rice's solution for the critical mode II stress intensity factor $K_{\mathrm{II}}$ at nucleation of a dislocation of edge character relative to the crack tip, for the $\theta=0$ case, is

$(1-v) K_{\mathrm{II}}{ }^{2} / 2 \mu=\gamma_{\text {us }}$

Here $\mu$ is the elastic shear modulus and $\nu$ is the Poisson ratio. The quantity on the left coincides with the energy release rate for pure mode II conditions and, since his analysis neglects tension-shear coupling along the slip plane, Rice's solution is insensitive to the mode I and mode III stress intensity factors in this $(\theta=0)$ case. A related solution, involving $K_{\mathrm{II}}$ and $K_{\mathrm{III}}$, was derived for nucleation of a general dislocation of mixed edge and screw character relative to the crack tip. An approximate solution of the same type of Peierls model for nucleation has been given by Schoeck [3]. The Peierls model has the advantage that the atomic properties which enter it are subject to direct estimation from atomic calculations. By contrast, the nucleation model of Rice and Thomson [4] and its descendents, based on elasticity solutions for fully formed dislocation lines or loops near a crack tip, require introduction of core cutoff procedures, so that the continuum elasticity problem and the atomic cut-off parameter which enters it are not as well defined.

Recently, there have been further studies of dislocation nucleation, and the related issue of ductile vs. brittle response, based on the Peierls framework. Sun et al. [5] used embedded atom method (EAM) models to estimate $\gamma_{\text {us }}$ for $\mathrm{Ni}$ and $\mathrm{Ni}_{3} \mathrm{Al}$ (see Foiles et al. [6] for $\mathrm{Ni}$, and Foiles and Daw [7] for $\mathrm{Ni}_{3} \mathrm{Al}$ for the origin of such models). Beltz and Rice [8] solved numerically the pair of integral equations describing the incipient dislocation for mixed mode loading of a crack with tension-shear coupling along a slip plane, in the simple case $\theta=0$ when the slip and crack plane coincide. They introduced a representation of the coupled tension-shear constitutive relations along the slip plane that we use here. Later, Beltz and Rice [9] applied similar procedures to numerical solution of cases with $\theta \neq 0$, for cracks along bimaterial (e.g. metal-ceramic) interfaces; related predictions of ductile $v s$. brittle response, based on that work and on ref. 10 were successfully tested experimentally for $\mathrm{Cu}$ crystals bonded to $\mathrm{Al}_{2} \mathrm{O}_{3}$ (sapphire) crystals by Beltz and Wang [11]. Rice et al. [12] review many of these developments, and give some preliminary results from EAM modeling of tension-shear coupling, as well as from consideration of elastic anisotropy. The latter topic is more fully treated by Sun et al. [13], whereas Beltz and Rice [14] give a first treatment within the Peierls type of integral equation modeling for the activation energy associated with dislocation nucleation.

The studies mentioned, as well as molecular dynamics simulations of crack tip dislocation nucleation in EAM Fe, and interpretation of the simulations, by Cheung [15] and Cheung et al. [16] have emphasized the importance of tension-shear coupling in easing the nucleation process. The effect comes about since opening displacements under tensile stress allow a slip process with less shear resistance. The implication of this tension softening effect for ductile $v$ s. brittle behavior was first discussed by Kelly et al. [17]. Argon [18] treated the tension effect by an effective shear modulus linearly weakened by tension.

\section{Integral equation formulation of elasticity problem}

\subsection{Integral equations defining dislocation emission from a crack tip, considering tension-shear coupling}

We consider the process of a dislocation nucleation on an inclined slip plane of angle $\theta$ with respect to the crack plane under combined $K_{\mathrm{I}}$ and $K_{\mathrm{II}}$ loading, as shown in Fig. 1. An incipient static distribution $\left\{\delta_{r}(s), \delta_{\theta}(s)\right\}$, of sliding and opening displacement discontinuities across the slip plane develops in response to small loading by the stress concentration at the crack tip. Here we treat only pure edge slip, i.e. there are no antiplane or screw displacement discontinuities. In a later section we discuss results when an antiplane slip $\delta_{z}(s)$, of screw type relative to the crack tip, is considered. We seek the condition under which, with an increase in loading, no further incipient static distribution exists, i.e. the solution becomes unstable so that afterwards a dislocation line of a finite edge component ener ${ }_{i} \sim a r_{i}{ }^{\prime}$ moves away from the crack tip until stopped by lat...e resistance, or some other barrier. 
As Fig. 1 illustrates, the incipient profile $\left\{\delta_{r}(s), \delta_{\theta}(s)\right\}$ is modeled here as a continuous distribution of infinitesimal dislocations having, at location $s$, a Burgers vector $\left[-\left(\mathrm{d} \delta_{r}(s) / \mathrm{d} s\right) \mathrm{d} s,-\left\langle\mathrm{d} \delta_{\theta}(s) / \mathrm{d} s\right\rangle \mathrm{d} s\right]$, which in turn exerts stresses $\sigma_{\theta i}(r)=g_{i j}(r, s ; \theta)$ $\left[-\left(\mathrm{d} \delta_{j}(s) / \mathrm{d} s\right) \mathrm{d} s\right]$ on a point $r$ along the slip plane, as calculated for a linear elastic medium. Here, $i, j=r, \theta$ and there is summation on repeated indices. Therefore, the functions $g_{i j}(r, s ; \theta)$ so defined can be obtained by interactions of a line dislocation with the crack tip in linear elastic medium. The function $g_{i j}(r, s ; \theta)$ may be extracted from Lin and Thomson [19] and is listed fully by Beltz $[20]$. When $\theta=0$, the functions reduce to $g_{i j}(r, s ; 0)=\mu(s / r)^{1 / 2} \delta_{i j} / 2 \pi(1-v)(r-s)$, where $\delta_{i j}=1$ when $i=j$ and 0 otherwise.

The unrelaxed resolved shear stress in the slip plane before emergence of the slip and opening profile is

$\sigma_{\theta r}{ }^{\prime}(r)=\frac{K_{\mathrm{Il}}^{\mathrm{def}}}{(2 \pi r)^{1 / 2}}=\frac{f_{\mathrm{I}}^{\tau}(\theta) K_{\mathrm{I}}+f_{\mathrm{II}}{ }^{r}(\theta) K_{\mathrm{II}}}{(2 \pi r)^{1 / 2}}$

where $f_{\mathrm{I}}{ }^{\top}(\theta)=\cos ^{2}(\theta / 2) \sin (\theta / 2)$ and $f_{\mathrm{II}}{ }^{\top}(\theta)=\cos (\theta / 2)$ $\left[1-3 \sin ^{2}(\theta / 2)\right]$. The unrelaxed tensile stress is

$\sigma_{\theta \theta}{ }^{\prime \prime}(r)=\frac{K_{1}{ }^{\mathrm{eff}}}{(2 \pi r)^{1 / 2}}=\frac{f_{1}^{\sigma}(\theta) K_{\mathrm{I}}+f_{\mathrm{II}}{ }^{\sigma}(\theta) K_{\mathrm{II}}}{(2 \pi r)^{1 / 2}}$

where $f_{\mathrm{I}}{ }^{\sigma}(\theta)=\cos ^{3}(\theta / 2)$ and $f_{\mathrm{II}}{ }^{\sigma}(\theta)=-3 \cos ^{2}(\theta / 2)$ $\sin (\theta / 2)$. These correspond to the classical elastic singular field.

The diverging stresses $\sigma_{\theta r}{ }^{0}$ and $\sigma_{\theta \theta}{ }^{0}$ are relaxed by emergence of an incipient dislocation core, described by the displacement discontinuity profile $\left\{\delta_{r}(s), \delta_{\theta}(\mathrm{s})\right\}$. We treat this as a combined shear and tension stress relief process in order to delineate the role which the normal stress plays in dislocation nucleation, although the model can, of course, also describe the Griffith decohesion which results when the unrelaxed tensile stress is much larger than the shear stress (and no other slip planes are available). The equations of equilibrium for shear and tension along the slip plane are thus

$$
\begin{aligned}
\tau\left[\delta_{r}\langle r\rangle, \delta_{\theta}(r)\right]= & \sigma_{\theta r}{ }^{\prime}(r)-\int_{0}^{\infty} g_{11}(r, s ; \theta) \frac{\mathrm{d} \delta_{r}(s)}{\mathrm{d} s} \mathrm{~d} s \\
& -\int_{0}^{\infty} g_{12}(r, s ; \theta) \frac{\mathrm{d} \delta_{\theta}(s)}{\mathrm{d} s} \mathrm{~d} s
\end{aligned}
$$

and

$$
\begin{aligned}
\sigma\left[\delta_{r}(r), \delta_{\theta}(r)\right]= & \sigma_{\theta \theta}{ }^{0}(\mathbf{r})-\int_{0}^{\infty} g_{21}(r, s ; \theta) \frac{\mathrm{d} \delta_{r}(s)}{\mathrm{d} s} \mathrm{~d} s \\
& -\int_{0}^{\infty} g_{22}(r, s ; \theta) \frac{\mathrm{d} \delta_{\theta}(s)}{\mathrm{d} s} \mathrm{~d} s
\end{aligned}
$$

where our treatment adopts the approximation of a half-plane crack in an infinite solid under remote $K_{\mathrm{I}}$, $K_{\mathrm{II}}$ loading. The terms $\tau\left[\delta_{r}(r), \delta_{\theta}(r)\right]$ and $\sigma\left[\delta_{r}(r), \delta_{\theta}(r)\right]$ are the shear and tensile stresses across the slip plane, and a potential $\Phi\left[\delta_{r}(r), \delta_{\theta}(r)\right]$ is assumed to exist such that

$\tau\left[\delta_{r}(r), \delta_{\theta}(r)\right]=\frac{\partial \Phi\left[\delta_{r}(r), \delta_{\theta}(r)\right]}{\partial \delta_{r}(r)}$

$\sigma\left[\delta_{r}(r), \delta_{\theta}(r)\right]=\frac{\partial \Phi\left[\delta_{r}(r), \delta_{\theta}(r)\right]}{\partial \delta_{\theta}(r)}$

Modeling of the potential from EAM models will be given in the next section. Equations (4), (5), (6) and (7) constitute a complete set of equations which can be solved simultaneously. The solutions may be obtained by numerical methods.

Following developments by Beltz $[20]$ and Beltz and Rice $[8,9]$, the system of eqns. $(4),(5),(6)$ and (7) is solved most efficiently by utilizing the interpolating points for the distribution $\left\{\delta_{r}(r), \delta_{\theta}(r)\right\}$ at the $N+1$ roots of the Chebychev polynomial of the second kind of order $N+1$ (see, for example, Erdogan [21] and Erdogan and Gupta [22]). The numerical procedure searches for the convergent configuration $\left\{\delta_{r}(r), \delta_{\theta}(r)\right\}$ via a Newton-Raphson technique. Increasing the loading gradually up to a point where no solutions can be found (just before the displacement increments increase rapidly with loading increments) we then assume that the slip profile has become unstable and denote the corresponding loading as the critical loading for dislocation nucleation. An equivalent but more rigorous approach to determining the instability point is described by Beltz and Rice [8]; it involves monitoring the Jacobian matrix $\mathbf{J}$ of the non-linear algebraic equations. The determinant of $\mathbf{J}$ rapidly decreases towards zero at the instability.

\subsection{Special case based on the shear-only approximation}

The role of normal stress in dislocation nucleation analyzed in the combined shear and tension model at a crack tip is compared and contrasted with the treatment of dislocation emission as a pure shear process, in which case we have a simpler set of equations to solve,

$\tau\left[\delta_{r}(r)\right]=\sigma_{\theta r}{ }^{\prime}(r)-\int_{0}^{\infty} g_{11}(r, s ; \theta) \frac{\mathrm{d} \delta_{r}(s)}{\mathrm{d} s} \mathrm{~d} s$

$\tau\left[\delta_{r}(r)\right]=\frac{\partial \Phi\left[\delta_{r}(r)\right]}{\partial \delta_{r}(r)}$

The angle $\theta$ is an important parameter for dislocation emission from a crack tip, and is determined by the available slip systems with respect to the crack 
plane. The angle $\theta$ determines the content of the kernel functions $g_{i j}(r, s, \theta)$ and the proportion of mixing of shear and tension, $K_{\mathrm{II}}{ }^{\text {eff }}$ and $K_{\mathrm{I}}^{\text {eff }}$.

For the case of a slip plane coplanar with the crack, $\theta=0$, Rice [1] showed that relations could be derived between the applied $K_{\mathrm{I}}, K_{\mathrm{II}}$ and the displacement discontinuities at the tip. These follow derivations like those of Rice [23, 24] and Eshelby [25] in applying the path-independent $J$-integral to cohesive crack models, or derivations in the style of Willis [26] based directly on the elasticity integral equations. The relations for the coupled tension-shear model of eqns. (4)-(7) are

$G \equiv \frac{1-v}{2 \mu}\left(K_{\mathrm{I}}^{2}+K_{\mathrm{lI}}^{2}\right)=\Phi\left(\delta_{r}^{\text {tip }}, \delta_{\theta}^{\text {tip }}\right)$

where $\delta_{i}^{\text {tip }}$ is $\delta_{i}(r)$ at $r=0$. Also, for the shear-only model described by eqns. $(8),(9)$,

$\frac{1-v}{2 \mu} K_{\mathrm{ll}}^{2}=\Phi\left(\delta_{r}^{\text {tip }}\right)$

Since $\gamma_{u s}$ is the maximum value of $\Phi(\delta)$ in the shearonly model, the above equation explains why the maximum loading at which an incipient dislocation exists, i.e. the nucleation loading, is given by eqn. (1). Since the model with tension-shear coupling involves two kinematic quantities, we cannot similarly use eqn. (10) to obtain the nucleation condition directly; the integral equations must actually be solved. We can, however, note that since $2 \gamma_{\mathrm{s}}\left(\gamma_{\mathrm{s}}\right.$ is the surface energy) is the maximum value of $\Phi$, attained as $\delta_{\theta}$ increases beyond the range of attraction of the two surfaces, then eqn. (10) assures that the Griffith loading $G=2 \gamma_{\mathrm{s}}$ gives an upper bound to the loading for dislocation nucleation in this case of coincident crack and slip planes.

There is some arbitrariness in how the potential $\Phi\left(\delta_{r}\right)$ for the shear-only approximation is chosen. Generally we can write

$\Phi\left(\delta_{r}\right)=\Phi\left(\delta_{r}, \delta_{\theta}=\right.$ constrained $)$

to indicate that different ways of constraining $\delta_{\theta}$ during the slip process lead to different functions $\Phi\left(\delta_{r}\right)$, and hence to different interpretations of its maximum value $\gamma_{u s}$. Some of these are discussed in the following sections.

\section{Combined tension-shear constitutive relations as constrained by atomic modeling}

\subsection{Form of a combined tension-shear constitutive law}

For a uniform slip process in a crystal lattice, we imagine that a plane splits the crystal into two blocks. The upper block translates by a displacement $\left(\Delta_{r}, \Delta_{\theta}\right)$

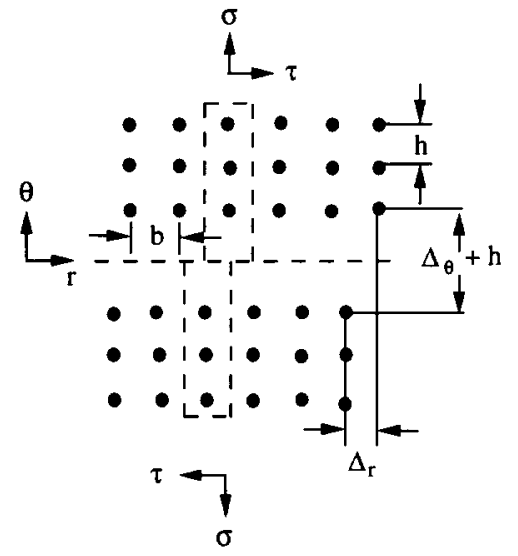

Fig. 2. Block-like lattice sliding and opening displacement of atomic layers along a slip plane. The atoms inside the column made of broken lines are counted for total energy summations.

relative to the lower block, as demonstrated in Fig. 2; $\Delta_{\theta}$ denotes an opening displacement and $\Delta_{r}$ is an in-plane shear. Consequently, the lattice is no longer periodic along the $\theta$ direction, but is still periodic along the $r$ and $z$ directions inside the plane. In this work, we only treat the relaxations associated with normal separation of the two blocks, which we believe to be the main relaxation effect in slip processes in elemental metals. Other modes of relaxation and their relevance to Peierls modeling are open issues for further research.

We may define a potential $\Psi\left(\Delta_{r}, \Delta_{\theta}\right)$ for the energy per unit area associated with displacement $\left(\Delta_{r}, \Delta_{\theta}\right)$. The work quantities conjugate to $\left(\Delta_{r}, \Delta_{\theta}\right)$ are the shear stress $\tau$ and normal stress $\sigma$ such that

$\mathrm{d} \Psi\left(\Delta_{r}, \Delta_{\theta}\right)=\tau \mathrm{d} \Delta_{r}+\sigma \mathrm{d} \Delta_{\theta}$

The potential denoted $\Phi\left(\delta_{r}, \delta_{\theta}\right)$ earlier involved the displacement discontinuities across the slip plane (a mathematical plane without thickness). The related potential denoted $\Psi\left(\Delta_{r}, \Delta_{\theta}\right)$ here is discussed for combined slip and opening displacements between the two atomic planes with initial normal separation $h$. We will give the transformation from the potential $\Psi\left(\Delta_{r}, \Delta_{\theta}\right)$ and relative atomic displacements $\left(\Delta_{r}, \Delta_{\theta}\right)$ to the potential $\Phi\left(\delta_{r}, \delta_{\theta}\right)$ and displacement discontinuities $\left(\delta_{r}, \delta_{\theta}\right)$ in the final part of this section.

In the unrelaxed shear mode, i.e. sliding by $\Delta_{r}$ with $\Delta_{\theta}=0$, the shear stress $\tau\left(\Delta_{r}\right)$ vs. the sliding displacement $\Delta_{r}$ is conveniently approximated by the sinusoidal form $[2,27]$,

$\tau\left(\Delta_{r}\right)=\left(\frac{\pi \gamma_{u s}{ }^{(u)}}{b}\right) \sin \left(\frac{2 \pi \Delta_{r}}{b}\right)$

where now the coefficient, which could also be written as $\mu b / 2 \pi h$, is given in terms of the unrelaxed value of the unstable stacking energy, where $h$ is the interlayer 
separation distance. Thus $\Psi\left(\Delta_{r}, \Delta_{\theta}=0\right)$ has a maximum $\gamma_{\text {us }}{ }^{\left({ }^{\prime}\right)}$ (unrelaxed value) at $\Delta_{r}=b / 2$.

However, for normal decohesion at $\Delta_{r}=0$, we may adopt the standard approximate form of the Rose-Ferrante-Smith [28] universal binding relation between tensile stress $\sigma\left(\Delta_{\theta}\right)$ and opening displacement $\Delta_{\theta}$, as found to fit the numerical solutions for decohesion based on density functional theory (DFT) in the local density approximation (LDA). This is

$\sigma\left(\Delta_{\theta}\right)=\left(\frac{2 \gamma_{\mathrm{s}}}{L}\right)\left(\Delta_{\theta} / L\right) \exp \left(-\Delta_{\theta} / L\right)$

where $\gamma_{\mathrm{s}}$ is the surface energy for one of the decohered surfaces; Rose et al. [28] report that the characteristic distance $L$ is found to scale among elemental metals like the Thomas-Fermi screening distance. Equation (15) is, via eqn. (13), consistent with the Rose-Ferrante-Smith [28] universal binding relation for the energy excess per unit area, as approximated in the form

$\Psi\left(\Delta_{r}=0, \Delta_{\theta}\right)=2 \gamma_{s}\left\{1-\left[1+\left(\Delta_{\theta} / L\right)\right] \exp \left(-\Delta_{\theta} / L\right)\right\}$

Now, to construct approximate constitutive relations for the combined shear and tension case, we assume $[8$, 12] that $\tau\left(\Delta_{r}, \Delta_{\theta}\right)$ and $\sigma\left(\Delta_{r}, \Delta_{\theta}\right)$ have the general mathematical forms which follow, consistent with periodicity $b$ in shear and with characteristic length $L$ in the decohesion process. These forms are

$\tau\left(\Delta_{r}, \Delta_{\theta}\right)=A\left(\Delta_{\theta}\right) \sin \left(\frac{2 \pi \Delta_{r}}{b}\right)$

$\sigma\left(\Delta_{r}, \Delta_{\theta}\right)=\left[B\left(\Delta_{r}\right)\left(\Delta_{\theta} / L\right)-C\left(\Delta_{r}\right)\right] \exp \left(-\Delta_{\theta} / L\right)$

where $A\left(\Delta_{\theta}\right), B\left(\Delta_{r}\right)$ and $C\left(\Delta_{r}\right)$ are functions of their arguments. These functions are constrained, in a way which introduces just one more material parameter, when we require consistency with eqns. (14) and (15) when either $\Delta_{\theta}=0$ or $\Delta_{r}=0$, and when we further require that $\sigma$ and $\tau$ be consistent with the existence of a potential $\Psi$ as in eqn. (13). The latter condition requires that the Maxwell reciprocal relation

$\frac{\partial \tau}{\partial \Delta_{\theta}}=\frac{\partial \sigma}{\partial \Delta_{r}}$

be satisfied. The resulting functions $A, B, C$, obtained so as to require $\tau$ to vanish as $\Delta_{\theta} \rightarrow \infty$, are

$A\left(\Delta_{\theta}\right)=\frac{\pi \gamma_{\mathrm{us}}{ }^{(\mathrm{u})}}{b}\left\{1+\left(\frac{1-p / q}{1-p}\right)\left(\Delta_{\theta} / L\right)\right\} \exp \left(-\Delta_{\theta} / L\right)$

$$
\begin{aligned}
& B\left(\Delta_{r}\right)=\frac{2 \gamma_{s}}{L}\left[1-\left(\frac{q-p}{1-p}\right) \sin ^{2}\left(\frac{\pi \Delta_{r}}{b}\right)\right] \\
& C\left(\Delta_{r}\right)=\frac{2 \gamma_{s}}{L}\left[\frac{p(1-q)}{1-p} \sin ^{2}\left(\frac{\pi \Delta_{r}}{b}\right)\right]
\end{aligned}
$$

Here

$q=\frac{\gamma_{\mathrm{us}}^{(\mathrm{u})}}{2 \gamma_{\mathrm{s}}} \quad$ and $\quad p=\frac{\Delta_{\theta}{ }^{*}}{L}$

where $\Delta_{\theta}{ }^{*}$ is the new material parameter allowed by these considerations, and is the relaxed opening displacement corresponding to tensile stress $\sigma=0$ when the shear displacement $\Delta_{r}=b / 2$, i.e. the value of $\Delta_{r}$ at which $\tau=0$ at unstable stacking. The parameter $p$ is called the dilation parameter. The two quantities $p$ and $q$ measure the importance of tension-shear coupling, as will be seen further.

We obtain the potential $\Psi\left(\Delta_{r}, \Delta_{\theta}\right)$ consistent with eqns. (17), (18), (19) and (20) as

$$
\begin{aligned}
\Psi\left(\Delta_{r}, \Delta_{\theta}\right)= & 2 \gamma_{s}\left\{1-\left[1+\left(\Delta_{\theta} / L\right)\right] \exp \left(-\Delta_{\theta} / L\right)\right. \\
& +\sin ^{2}\left(\pi \Delta_{r} / b\right)\left[q+\left(\frac{q-p}{1-p}\right)\left(\Delta_{\theta} / L\right)\right] \\
& \left.\times \exp \left(-\Delta_{\theta} / L\right)\right\}
\end{aligned}
$$

and this reduces to a potential introduced by Needle$\operatorname{man}[29]$ when $p=q$.

The relaxed $\gamma_{u s}$ value $\gamma_{u s}{ }_{u s}^{(r)}$ which is the energy $\Psi$ at equilibrium in the unstable stacking configuration requiring no stresses $(\tau=0$ and $\sigma=0)$, is obtainable from eqn. (22) by setting the shear displacement $\Delta_{r}=b / 2$ and the opening displacement $\Delta_{\theta}=\Delta_{\theta}{ }^{*}$, i.e. setting $\Delta_{\theta} / L=p$. Omitting the algebra, we arrive at the relation,

$\frac{\gamma_{\mathrm{us}}^{(\mathrm{r})}-\gamma_{\mathrm{us}}^{(\mathrm{u})}}{\gamma_{\mathrm{us}}^{(\mathrm{u})}}=-\left[\frac{1-q}{q(1-p)}\right][\exp (-p)-1+p]$

\subsection{Results from $E A M$ calculations for models of $F e$, Al and $\mathrm{Ni}$}

The atomic calculations for embedded atom method $\mathrm{Fe}, \mathrm{Al}, \mathrm{Ni}$ and $\mathrm{Ni}_{3} \mathrm{Al}$ were performed in the following way. The energy calculations involve double summations. The EAM functions are usually cut off at a distance $r_{\text {cut }}$ which is between the third and fourth nearest neighbor distances. Because of the cut-off we need to sum over the fourth nearest neighbors. 
We want to calculate energies associated with the slip process with coupling to opening displacement. The model is shown schematically in Fig. 2 . It is convenient to use triclinic axes $\left\{\boldsymbol{a}_{1}, \boldsymbol{a}_{2}, \boldsymbol{a}_{3}\right\}$, which can be chosen for the slip system and lattice type, for describing a lattice undergoing sliding and opening decohesion, with $\boldsymbol{a}_{1}$ and $\boldsymbol{a}_{2}$ marking the periodic lattice in the slip plane, and a third axis $a_{3}$ directed out of the slip plane.

The slip is designated as some fraction of the Burgers vector $\boldsymbol{b}$ expressed in some components on $\left\{\boldsymbol{a}_{1}, \boldsymbol{a}_{2}\right\}$. Define $\boldsymbol{e}_{z}$ as a vector in the slip plane and perpendicular to the Burgers vector, and $\boldsymbol{e}_{\theta}$ a vector perpendicular to the slip plane having components in $\left\{a_{1}, a_{2}, a_{3}\right\}$. The original lattice is composed by putting atoms on all space points, spanned by integer numbers as the coordinates in the basis $\left\{\boldsymbol{a}_{1}, \boldsymbol{a}_{2}, \boldsymbol{a}_{3}\right\}$. So we let the upper block be displaced by a vector $\left(\Delta_{r}, \Delta_{\theta}, \Delta_{z}\right)$ in the basis of $\left\{\boldsymbol{b}, \boldsymbol{e}_{\theta}, \boldsymbol{e}_{z}\right\}$ respectively. The sliding routes are shown from the top view for a b.c.c. lattice in Fig. 3, the Shockley partial route in an f.c.c. lattice in Fig. 6, and for $\mathrm{Ni}_{3} \mathrm{Al}$ in Fig. 11.

The configuration after sliding and opening displacements is still periodic inside the two-dimensional plane, the sliding plane spanned by basis $\left\{a_{1}, a_{2}\right\}$. However, it is no longer periodic along the $a_{3}$ direction, pointing out of the sliding plane (Fig. 2 shows that). This suggests the way in which we choose atoms so that the energy excess from such displacements can be calculated. In the direction normal to the slip planes, we put a few extra layers on top of the upper block and also below the lower block to provide the necessary embedding medium, usually three layers so that the embedding electronic density contribution for the blocks from the boundary layers and the pairwise interactions between the boundary layers and the blocks are all taken care of. When a slip is imposed on the two blocks, we let the boundary layers also move with their associated block. Hence, the way our calculations of the energy of a generalized stacking fault are performed does not involve either free boundaries or a periodic array of stacking faults along the normal direction.

The atoms inside a column are chosen, shown in Fig. 2 , in order to be counted in summing for the total energy: we start by choosing a unit cell in the basis of $\left\{a_{1}, a_{2}, a_{3}\right\}$, then let the unit cell repeat along the $\boldsymbol{a}_{3}$ direction both above and below the sliding plane for the same number of units in $a_{3}$. The upper and bottom blocks should be as thick as twice the cut-off distance to calculate the energy accurately. The atoms will interact both with the atoms inside the column and with those outside, including atoms in the boundary layers, as long as they are within the interacting range. Trial calculations showed we only need to include, for the total energy calculations, five units along $a_{3}$ directions each for both blocks above and below the slip plane, in order to yield unchanging results when including more units along the $a_{3}$ direction.

We calculate the energy of the column in the fashion of direct numerical summations. We take the energy difference of the slipped configuration relative to the undisplaced configuration. Since the energy excess obviously scales with the area of the slip plane, we can define a potential energy associated with such a displacement $\Psi\left(\Delta_{r}, \Delta_{\theta}, \Delta_{z}\right)$ for the excess in energy per unit area. The calculations are always to evaluate the energy of the configuration with imposed sliding and opening condition. They should not be thought of as a type of simulation where a boundary condition is imposed and the system of atoms responds to it.

We now present results on the energy surface $\Psi\left(\Delta_{r}, \Delta_{\theta}\right)$ for sliding and opening between a pair of lattice slip planes as calculated based on EAM potentials intended to simulate approximately $\mathrm{Fe}, \mathrm{Al}$ and $\mathrm{Ni}$.

The slip route $(1 / 2)[111](011)$ in b.c.c. is shown in Fig. 3. Figure 4(a) shows the energy $\Psi\left(\Delta_{r}, \Delta_{\theta}\right)$ of EAM Fe (for the source of EAM potential, see Harrison et al. [30]) as a function of the slip displacement $\Delta_{r}$ at various opening displacements $\Delta_{\theta}$ for the slip system $(1 / 2)[111](011)$. With a finite amount of opening, the energy height in the path from zero slip displacement to that of a half Burgers vector is lowered, which is the source of tension softening in a shear process in the description within the Peierls framework, noted on the basis of molecular dynamics simulations based on this same potential by Cheung [15] and Cheung et al. [16]. Figure 4(b) shows the energy as a function of the decohesion $\Delta_{\theta}$ with slip displacement as a parameter. The analytical model of eqn. (22) describes well the general shape of the curves when we choose $L / b=0.197, q=0.158$ and $p=0.214$ (and $h / b$ from the b.c.c. lattice geometry is $\sqrt{2 / 3}$ ). The minimum energy point, with opening displacement as the variable, shifts with different slip displacements, which is accounted for in the analytical formula. Figure 5 compares the relaxed and unrelaxed energy vs. slip relations as calculated from the EAM model.

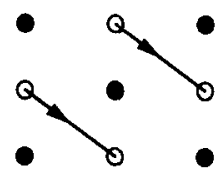

$\operatorname{slip}(1 / 2)\left[\begin{array}{lll}1 & 1 & 1\end{array}\right]\left(\begin{array}{lll}0 & -1 & 1\end{array}\right)$ in bcc

Fig. 3. The $(1 / 2)[111](011)$ slip in a b.c.c. lattice, top view. The empty and filled circles depict atoms adjacent to the slip plane on opposite sides. 
$\mathrm{Fe}(1 / 2)\left[\begin{array}{lll}1 & 1 & 1\end{array}\right]\left(\begin{array}{lll}0 & -1 & 1\end{array}\right)$
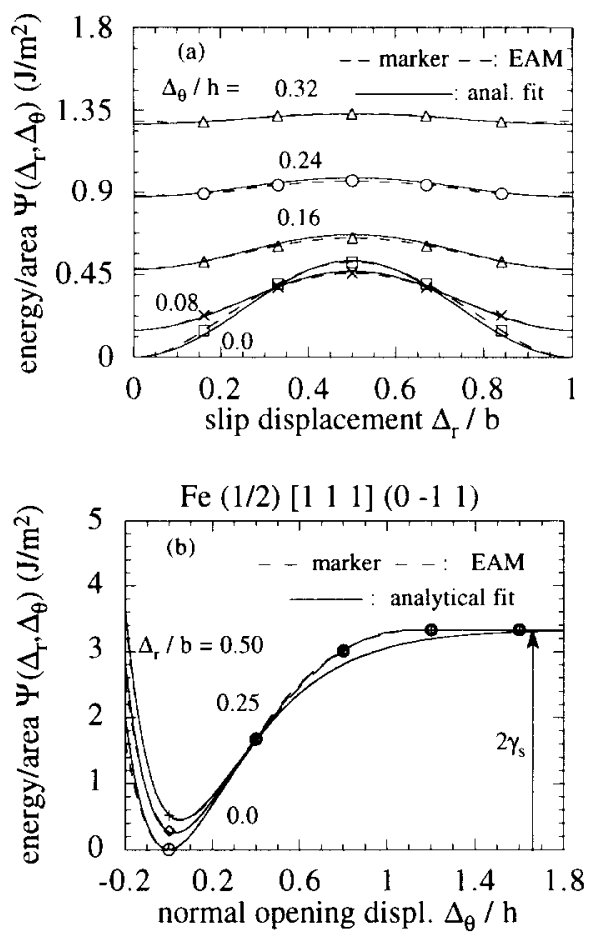

Fig. 4. The potential energy $\Psi\left(\Delta_{r}, \Delta_{\theta}\right)$, (a) as a function of the sliding displacement $\Delta_{r}$ and (b) as a function of opening displacement $\Delta_{\theta}$, for the $\mathrm{Fe}(1 / 2)[111](0 \overline{1} 1)$ slip system. Broken curves with markers are obtained from atomistic calculations based on the embedded atom method; solid curves are from modeling based on the analytical formula, eqn. (22).

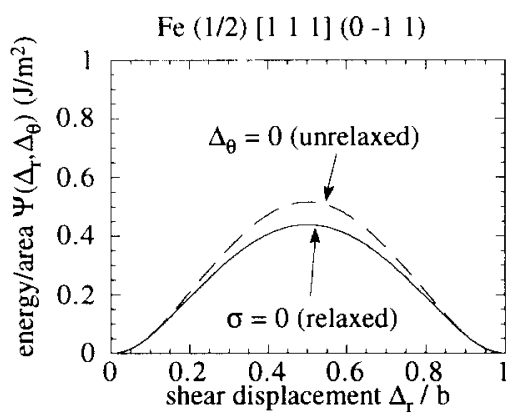

Fig. 5. The potential energy $\Psi\left(\Delta_{r}, \Delta_{\theta}\right)$ of $(1 / 2)[111](011)$ slip for Fe along the constrained path $\left(\Delta_{\theta}=0\right)$ and relaxed path $\left(\Delta_{\theta}\right.$ relaxed to value to minimize the energy $\Psi$, i.e. to make $\sigma=0$ ) from atomistic calculations based on the embedded atom method.

Based on the similarity of the EAM curves (broken lines) and the modeling curves (solid lines), obtained from eqn. (22), in Fig. 4, we see that the analytical formula expressed in eqn. (22) is satisfactory for describing the tension-shear coupled constitutive law for combined sliding and opening displacements. Therefore, we use the analytical formula in the numeri-

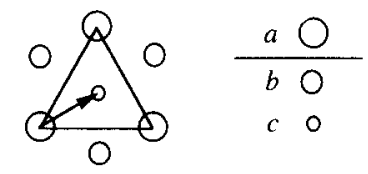

Shockley partial slip, (1/6) [ $\left[\begin{array}{lll}-2 & 1 & 1\end{array}\right]$ (111) in fcc

Fig. 6. The $(1 / 6)[\overline{2} 11](111)$ slip, i.e. Shockley partial route, in an f.c.c. lattice, top view. The large, medium and small circles depict atoms of layers $a, b$ and $c$. The slip plane is between the layers a and $b$.

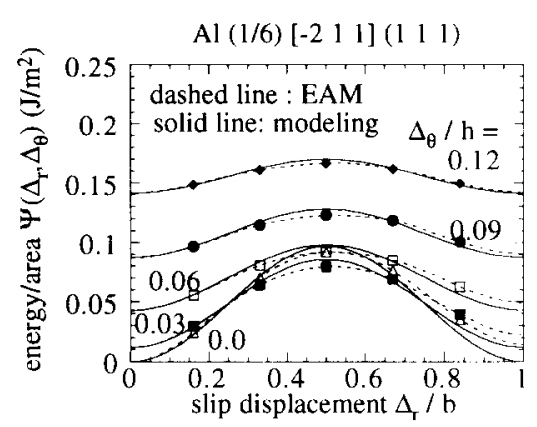

Fig. 7. The energy $\Psi\left(\Delta_{r}, \Delta_{\theta}\right)$ as a function of the shear displacement $\Delta_{r}$ and opening displacement $\Delta_{\theta}$ for the $\mathrm{Al}(1 / 6)[\overline{2} 11](111)$ slip system from atomistic calculations based on the embedded atom method (-- ). Results of analytical fitting using eqn. (22) $(-)$ are also shown.

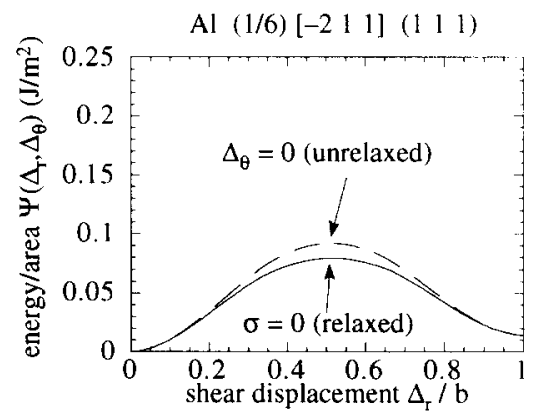

Fig. 8. The potential energy $\Psi\left(\Delta_{r}, \Delta_{\theta}\right)$ of $(1 / 6)[\overline{2} 11](111)$ slip for Al along the constrained path $\left(\Delta_{\theta}=0\right)$ and relaxed path $\left(\Delta_{\theta}\right.$ relaxed to value to minimize the energy $\Psi$ ) from atomistic calculations based on the embedded atom method.

cal formulation of the integral equations describing dislocation emission.

The slip $(1 / 6)[\overline{2} 11](111)$ in an f.c.c. solid, i.e. the Shockley partial route, is shown in Fig. 6. Figure 7 shows the energy $\Psi\left(\Delta_{r}, \Delta_{\theta}\right)$ as a function of the slip displacement $\Delta_{r}$ at various opening displacements $\Delta_{\theta}$ for $\mathrm{Al}$ (for the source of the potential, see Hoagland $e t$ al. [31]), while Fig. 8 shows it when the normal separation distance between the two blocks is relaxed or 
unrelaxed. Figures 9 and 10 show similar curves for EAM Ni (for the source of the potential, see Foiles et al. [6]). Note the decrease in unstable stacking energy when the normal separation is relaxed, shown in Figs. $5,8,10$ and 12 .

In the f.c.c. cases, $\Psi$ does not vanish when the lattice is shifted by $\Delta_{r}=b$ and relaxed in the normal direction (see right sides of Figs. 7-10). That is because $b$ corresponds to a partial dislocation, and the residual value of $\Psi$ corresponds to the stacking fault energy $\gamma_{\mathrm{sf}}$. That parameter is, in fact, not very well described by the EAM models; Hirth and Lothe [32] give $\gamma_{\mathrm{sf}}=125 \mathrm{~mJ}$ $\mathrm{m}^{-2}$ for Ni whereas the EAM model predicts $\gamma_{\mathrm{sf}}=14.5$

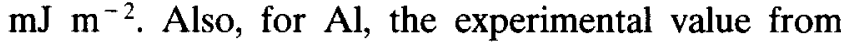
Hirth and Lothe [32] is $\gamma_{\mathrm{sf}}=166 \mathrm{~mJ} \mathrm{~m}^{-2}$ while EAM gives $\gamma_{\mathrm{sf}}=14.1 \mathrm{~mJ} \mathrm{~m}^{-2}$. In our fit to the analytical potential of eqn. (22), we focus on the EAM results up to the peak in $\Psi$ at $\Delta_{r} \approx b / 2$, since that is the part of the potential experienced in the Peierls modeling prior to the nucleation instability. The maximum of $\Psi$ at fixed $\Delta_{\theta}$ is still very close to $\Delta_{r}=b / 2$.

The fitting is done as follows. The value of $2 \gamma_{\mathrm{s}}$ is fixed at the EAM value, i.e. the leveling-off value of $\Psi$

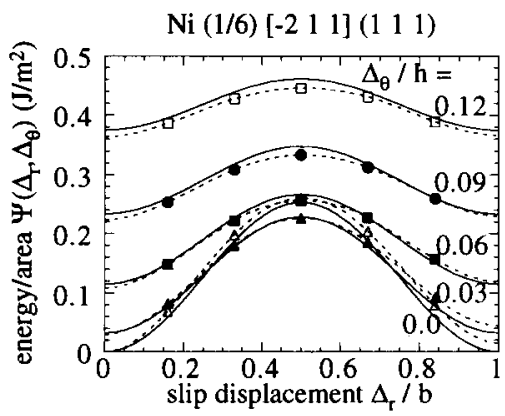

Fig. 9. The energy $\Psi\left(\Delta_{r}, \Delta_{\theta}\right)$ surface as function of the shear displacement $\Delta_{r}$ and opening displacement $\Delta_{\theta}$ for the $\mathrm{Ni}(1 / 6)$ $[211](111)$ slip system from atomistic calculations based on the embedded atom method (-- ). Results of analytical fitting using eqn. $(22)(-)$ are also shown.

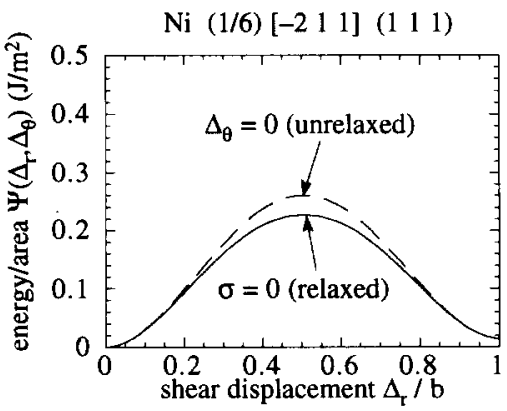

Fig. 10. The potential energy $\Psi\left(\Delta_{r}, \Delta_{\theta}\right)$ surface of $(1 / 6)$ [211](111) slip for $\mathrm{Ni}$ along the constrained path $\left(\Delta_{\theta}=0\right)$ and relaxed path $\left(\Delta_{\theta}\right.$ relaxed to value to minimize the energy $\left.\Psi\right)$ from atomistic calculations based on the embedded atom method. at large opening $\Delta_{\theta}$. The set of parameters $q, p$ and $L / b$ is chosen in a non-linear fitting procedure [33] so that the analytical function of eqn. (22) gives the best fit in the least square sense to the EAM results for $\Psi\left(\Delta_{r}, \Delta_{\theta}\right)$. That is, we choose the set of parameters $q, p$ and $L / b$ to minimize the sum of squares of differences of the energy $\Psi\left(\Delta_{r}, \Delta_{\theta}\right)$ from the EAM computations and that obtained from the analytical formula at each grid point in a mesh of the two variables $\left(\Delta_{r}, \Delta_{\theta}\right)$. We would prefer the analytical formula to describe the energy function $\Psi\left(\Delta_{r}, \Delta_{\theta}\right)$ from the EAM computations in as wide a range as possible, starting with the most relevant range for dislocation nucleation. We have used slightly different fitting ranges for these four EAM materials as follows: For Fe, 0 to $b$ in 100 divisions for $\Delta_{r},-0.14 h$ to $0.24 h$ in 38 divisions for $\Delta_{\theta}$; for $\mathrm{Ni}, 0$ to $b$ in 100 divisions for $\Delta_{r},-0.105 h$ to $0.300 h$ in 27 divisions for $\Delta_{\theta}$; for $\mathrm{Al}, 0$ to $b$ in 100 divisions for $\Delta r,-0.12 h$ to $0.255 h$ in 25 divisions for $\Delta_{\theta}$; for $\mathrm{Ni}_{3} \mathrm{Al}$ (see below), 0 to $b\left(b\right.$ here is $\left.a_{0} / \sqrt{6}\right)$ in 100 divisions for $\Delta_{r},-0.03 h$ to $0.18 h$ in 7 divisions for $\Delta_{\theta}$. The fitting results in the most relevant range are shown with the EAM results in Figs. 4 for $\mathrm{Fe}, 7$ for $\mathrm{Al}$ and 9 for $\mathrm{Ni}$. When we solve for the critical conditions for dislocation nucleation at a crack tip, we know that values near $0.6 b$ for $\Delta_{r}$ are sometimes involved, but not too much beyond that value. Therefore, we need to be concerned with the quality of the analytical representation for values of $\Delta_{r}$ from 0 to slightly beyond $0.5 b$, in which range the analytical fitting by eqn. (22) for these three materials is satisfactory. We actually include the whole range from 0 to $b$ for $\Delta_{r}$ in the fitting, but the closeness of fit beyond $0.6 b$ is less important than the range from 0 to $0.6 b$ for $\Delta r$.

Table 1 summarizes the parameters of the combined shear and tension constitutive law for these EAM metals, which were obtained by fitting the analytical formula, eqn. (22) to the numerical results by atomistic calculations using EAM potentials. Properties determined directly from the EAM calculations, rather than the fitting procedure, are shown in Table 2. Some of the ratios of $\gamma_{\mathrm{us}}$ values shown in Table 2 are also shown in Table 3 based on properties from the fitting procedure, i.e. from Table 1 .

\subsection{Results from $\mathrm{EAM}$ calculations for $\mathrm{Ni}_{3} \mathrm{Al}$}

The route to a full slip on a (111) plane in $\mathrm{Ni}_{3} \mathrm{Al}$ can be viewed in Fig. 11. The easiest route is $O \rightarrow M \rightarrow R \rightarrow M^{\prime} \rightarrow O^{\prime}$. The associated energy of the lattice for sliding along the route is calculated with the EAM functions, taken from Foiles and Daw [7]. For the whole route, the relaxed energy (the solid curves), i.e. with the condition that the normal tension is zero, is shown in Fig. 12 alongside the unrelaxed energy (the broken curves), i.e. with the constraint of no normal 
TABLE 1. Properties of the analytical combined shear-tension law of eqn. (22) as fitted to results of atomistic calculations for the EAM Fe, Al, $\mathrm{Ni}$ and $\mathrm{Ni}_{3} \mathrm{Al}$, and DFT-LDA Si (glide and shuffle set)

\begin{tabular}{|c|c|c|c|c|c|c|}
\hline Material and slip system & \multicolumn{2}{|c|}{$\gamma_{\mathrm{us}}^{(\mathrm{r})}\left(\mathbf{J ~ m}^{-2}\right)^{\left(\gamma_{\mathrm{us}}{ }^{(\mathrm{u})}\right)}$} & \multirow{2}{*}{$\frac{\begin{array}{l}2 \gamma_{\mathrm{s}} \\
\left(\mathrm{J} \mathrm{m}^{-2}\right)\end{array}}{3.33}$} & \multirow{2}{*}{$\begin{array}{l}p \\
0.214\end{array}$} & \multirow{2}{*}{$\begin{array}{l}q \\
0.158\end{array}$} & \multirow{2}{*}{$\begin{array}{l}L / b \\
0.197\end{array}$} \\
\hline $\mathrm{Fe}(1 / 2)[111](1 \overline{1} 0)$ & 0.438 & $(0.517)$ & & & & \\
\hline $\mathrm{Al}(1 / 6)[211](111)$ & 0.0795 & $(0.0920)$ & 1.13 & 0.140 & 0.0854 & 0.279 \\
\hline $\mathrm{Ni}(1 / 6)[\overline{2} 11](111)$ & 0.226 & $(0.260)$ & 2.87 & 0.132 & 0.0879 & 0.271 \\
\hline $\mathrm{Ni}_{3} \mathrm{Al}(1 / 6)[211](111)^{\mathrm{a}}$ & 0.316 & $(0.348)$ & 3.30 & 0.150 & 0.108 & 0.256 \\
\hline Si glide $(1 / 6)[\overline{2} 11](111)^{b}$ & 1.91 & $(2.02)$ & 3.12 & 0.376 & 0.647 & 0.308 \\
\hline Si glide $(1 / 6)[211](111)^{c}$ & 1.91 & $(2.02)$ & 3.12 & 0.747 & 0.647 & 0.155 \\
\hline Si shuffle $(1 / 2)[011](111)^{b}$ & 1.67 & (1.81) & 2.68 & -0.650 & 0.675 & 0.121 \\
\hline Si shuffle $(1 / 2)[011](111)^{c}$ & 1.67 & $(1.81)$ & 2.68 & $-0.881^{\mathrm{d}}$ & 0.675 & 0.090 \\
\hline
\end{tabular}

${ }^{\mathrm{a}}$ Here the Burgers vector $b$ is also a fitting parameter, set equal to $1.18 a_{0} / \sqrt{6}$.

${ }^{\mathrm{b}} p, L$ are determined from the ratio $\gamma_{\mathrm{us}}{ }^{(\mathrm{r})} / \gamma_{\mathrm{us}}{ }^{(\mathrm{u})}$.

${ }^{c} L$ is given by Rose et al. [34].

dThere is a problem that $p^{2}>q$, which is not allowed, for this set of estimates.

TABLE 2. The properties as calculated directly from EAM for $\mathrm{Fe}, \mathrm{Al}, \mathrm{Ni}$ and $\mathrm{Ni}_{3} \mathrm{Al}$

\begin{tabular}{|c|c|c|c|c|c|c|}
\hline Material and slip system & $\gamma_{\mathrm{us}}^{(\mathrm{r})}$ & $\begin{array}{l}\gamma_{\mathbf{u s}}(\mathbf{u}\} \\
\left(\mathbf{J} \mathbf{m}^{-2}\right)\end{array}$ & $\begin{array}{l}\gamma_{\mathrm{us}^{\left(\mathrm{u}^{*}\right)}} \\
\left(\mathrm{J} \mathrm{m}^{-2}\right)\end{array}$ & $\gamma_{\mathrm{us}}{ }^{(\mathrm{r})} / \gamma_{\mathrm{us}}{ }^{(\mathrm{u})}$ & $\gamma_{\mathrm{us}}^{\left(\mathrm{u}^{*}\right)} / \gamma_{\mathrm{us}}{ }^{(\mathrm{u})}$ & $\gamma_{\text {us }}{ }^{\left(\mathrm{u}^{*}\right\rangle} / \gamma_{\mathrm{us}}{ }^{\langle\mathrm{r}}$ \\
\hline $\mathrm{Fe}(1 / 2)[111](1 \mathrm{1} 0)$ & 0.438 & 0.517 & 0.373 & 0.847 & 0.722 & 0.852 \\
\hline $\mathrm{Al}(1 / 6)[\overline{2} 11](111)$ & 0.0795 & 0.0920 & 0.0689 & 0.864 & 0.749 & 0.867 \\
\hline $\mathrm{Ni}(1 / 6)[\overline{2} 11](111)$ & 0.226 & 0.260 & 0.198 & 0.869 & 0.762 & 0.876 \\
\hline $\mathrm{Ni}_{3} \mathrm{Al}(1 / 6)[211](111)$ & 0.316 & 0.348 & 0.287 & 0.907 & 0.825 & 0.910 \\
\hline
\end{tabular}

TABLE 3. The properties as calculated from the parametrized combined shear-tension law for the $\mathrm{EAM} \mathrm{Fe}, \mathrm{Al}, \mathrm{Ni}$ and $\mathrm{Ni}{ }_{3} \mathrm{Al}$, and DFT-LDA Si glide and shuffle set

\begin{tabular}{|c|c|c|c|c|}
\hline Material and slip system & $p, q$ & $\gamma_{\text {us }}^{(r)} / \gamma_{\text {us }}^{(u)}$ & $\gamma_{u s}{ }^{\left(u^{*}\right)} / \gamma_{u s}{ }^{(u)}$ & $\gamma_{\mathrm{us}}^{\left(\mathrm{us}^{*}\right)} / \gamma_{\mathrm{us}}^{(\mathrm{r})}$ \\
\hline $\mathrm{Fe}(1 / 2)[111](1 \overline{1} 0)$ & $0.214,0.158$ & 0.855 & 0.729 & 0.853 \\
\hline $\mathrm{Al}(1 / 6)[211](111)$ & $0.140,0.0854$ & 0.882 & 0.779 & 0.881 \\
\hline $\mathrm{Ni}(1 / 6)[\overline{2} 11](111)$ & $0.132,0.0879$ & 0.900 & 0.810 & 0.899 \\
\hline $\mathrm{Ni}_{3} \mathrm{Al}(1 / 6)[211](111)$ & $0.150,0.108$ & 0.896 & 0.802 & 0.895 \\
\hline $\mathrm{Si}(1 / 6)[211](111)$ (glide) & $0.376,0.647$ & 0.945 & 0.860 & 0.910 \\
\hline $\mathrm{Si}(1 / 2)[110](111)$ (shuffle) & $-0.650,0.675$ & 0.923 & 0.434 & 0.471 \\
\hline
\end{tabular}

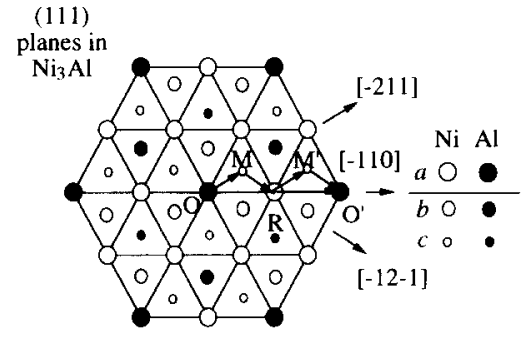

Fig. 11. The $\mathrm{Ni}_{3} \mathrm{Al}(111)$ planes. Open and filled circles represent the $\mathrm{Ni}$ and $\mathrm{Al}$ atoms respectively. Large, medium and small circles represent the $\mathrm{a}, \mathrm{b}$ and $\mathrm{c}$ plane layers respectively.

opening, which has been given in ref. 5. The estimates of the relaxed complex stacking fault (CSF) energy and relaxed (111) antiphase boundary (APB) energy are
$0.273 \mathrm{~J} \mathrm{~m}^{-2}$ and $0.198 \mathrm{~J} \mathrm{~m}^{-2}$ respectively. The (111) $\mathrm{APB}$ energy for $\mathrm{Ni}_{3} \mathrm{Al}$ using the same version of the EAM functions was calculated by Foiles to be 0.156 $\mathrm{J} \mathrm{m}^{-2}$ [35], with individual atomic relaxation. The relaxed unstable stacking energies are estimated to be $0.315 \mathrm{~J} \mathrm{~m}^{-2}$ for the first (also the same as for the fourth) encountered barrier, and $0.501 \mathrm{~J} \mathrm{~m}^{-2}$ for the second (also the same as for the third), while the corresponding unrelaxed values are 0.348 and $0.575 \mathrm{~J} \mathrm{~m}^{-2}$.

Of importance is the sliding path over the first barrier to a stable stacking fault, the CSF. We would like to represent the EAM curves by the analytical formula for the energy $\Psi\left(\Delta_{r}, \Delta_{\theta}\right)$ of the tensionshear coupled model, eqn. (22). Notice that the peak value is at a sliding that is somewhat greater than $b / 2$, had we taken $b$ to be $a_{0} / \sqrt{6}$ for the Shockley par- 


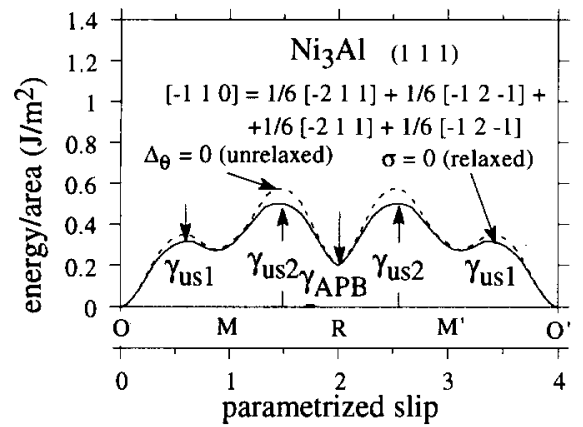

Fig. 12. The energy of a generalized stacking fault on a (111) plane in $\mathrm{Ni}_{3} \mathrm{Al}$ as a function of the slip distance in the Shockley partial route. The solid curve corresponds to the relaxed case where $\sigma=0$, while the dashed curve corresponds to the unrelaxed case $\Delta_{\theta}=0$.
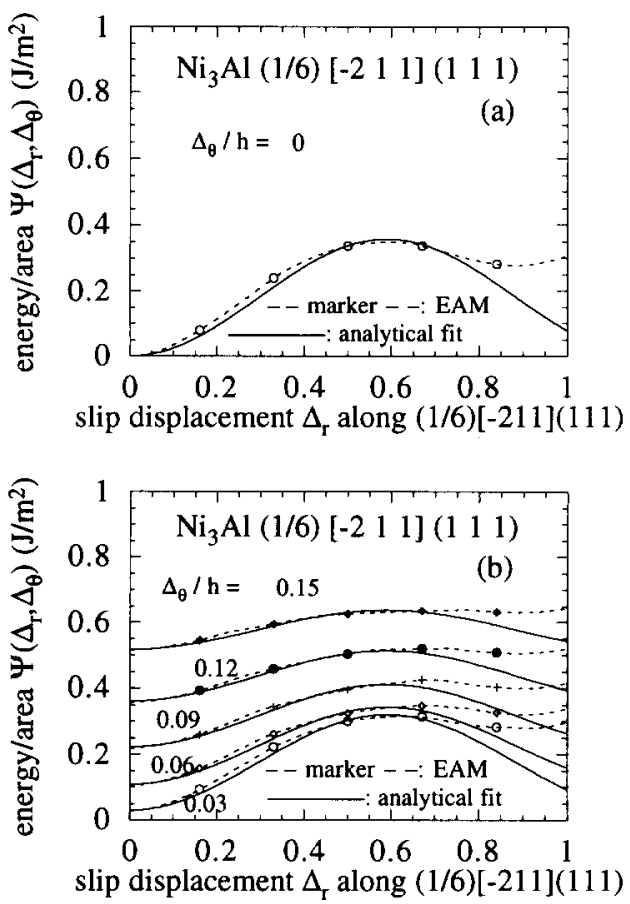

Fig. 13. The energy $\Psi\left(\Delta_{r}, \Delta_{\theta}\right)$ surface as a function of the shear displacement $\Delta_{r}$ for the $\mathrm{Ni}_{3} \mathrm{Al}(1 / 6)[211](111)$ slip system from atomistic calculations based on the embedded atom method $(---)$. Results of fitting using eqn. (22) (_-) are also shown. (a) Basis for choosing $b$, slightly larger than $a_{0} / \sqrt{6}$, (b) results over a wide range.

tial in the f.c.c. structure. We performed the fitting by taking the maximum point in the unrelaxed curve to be at $b / 2$ and defined $b$ this way; see Fig. 13(a). We found $b$ to be $(1.18)\left(a_{0} / \sqrt{6}\right)=0.172 \mathrm{~nm}$. The value $2 \gamma_{\mathrm{s}}$ for the cleavage energy is fixed at the EAM result of $3.30 \mathrm{~J}$ $\mathrm{m}^{-2}$. The fitting done this way gives $q=0.108$, $p=0.150$ and $L / b=0.256$. Note that $b$ here is also a fitting parameter. The EAM and analytical fitting results are shown in Fig. 13.
The set of parameters $\left(q, p, L / b, b, 2 \gamma_{\mathrm{s}}\right)$, as entered in Table 1, for overcoming the first barrier, for nucleating the first Shockley partial in $\mathrm{Ni}_{3} \mathrm{Al}$, does not carry over exactly for the tension-shear coupling involved in overcoming the other barriers.

\subsection{Results for Si from density functional theory}

Recently, Kaxiras and Duesbery [36] have reported similar calculations done by DFT-LDA, for shearing of Si along (111) planes. They studied both unrelaxed and relaxed configurations, in the sense of block-like lattice motions as in Fig. 2, in the vicinity of the unstable stacking configuration. Further, their studies of configurations that were unrelaxed in the block-like sense (i.e. corresponding to $\Delta_{\theta}=0$ here) were, nevertheless, locally relaxed relative to atomic positions in the opening direction for the four lattice planes immediately bordering the sliding plane. An earlier version of some of these results was reported by Duesbery et al. [37], corresponding to $\Delta_{\theta}=0 \cdot$ but with no local relaxations of atoms bordering the slip plane.

For $\mathrm{Si}$ and other materials of diamond-cubic structure, two types of (111) planes are of interest. These are the shuffle plane, which cuts through single covalent bonds along the direction perpendicular to the (111) plane, and the glide plane, which cuts through triplets of covalent bonds which are inclined equally to the (111) plane. For a partial dislocation along a $\{111\}$ glide plane, corresponding to a slip in a $\langle 211\rangle$ direction, Kaxiras and Duesbery [36] find $\gamma_{\mathrm{us}}^{(\mathrm{u})}=2.02 \mathrm{~J} \mathrm{~m}^{-2}$, $\gamma_{\mathrm{us}}{ }^{(\mathrm{r})}=1.91 \mathrm{~J} \mathrm{~m}^{-2}$ for $\mathrm{Si}$. Their results also give $\Delta_{\theta}{ }^{*}=0.0257 \mathrm{~nm}$. For introduction of a full $\langle 110\rangle$ dislocation along the $\{111\}$ shuffle plane, the corresponding results are $\gamma_{\mathrm{us}}{ }^{(\mathrm{u})}=1.81 \mathrm{~J} \mathrm{~m}^{-2}, \gamma_{\mathrm{us}}{ }^{(\mathrm{r})}=1.67 \mathrm{~J} \mathrm{~m}^{-2}$ and $\Delta_{\theta}{ }^{*}=-0.0303 \mathrm{~nm}$. Additional parameters of importance for Si are $b=0.222 \mathrm{~nm}$ for the partial glide plane dislocation and $b=0.384 \mathrm{~nm}$ for a full dislocation on either plane.

Huang et al. [38] used similar DFT-LDA methods to calculate surface energies for (111) planes of $\mathrm{Si}$. For the glide plane, the result is $2 \gamma_{\mathrm{s}}=3.12 \mathrm{~J} \mathrm{~m}^{-2}$ after reconstruction, and $2 \gamma_{\mathrm{s}}=3.21 \mathrm{~J} \mathrm{~m}^{-2}$ when constrained against reconstruction [39]. For the (111) shuffle plane, the result is $2 \gamma_{\mathrm{s}}=2.68 \mathrm{~J} \mathrm{~m}^{-2}$ with reconstruction.

There is no direct source in the DFT-LDA results, as reported, for our parameter $L$. One estimate is $L \approx 0.0344 \mathrm{~nm}$ from the Rose et al. [34] correlation of $L$ with the Thomas-Fermi screening length. Then in describing $\mathrm{Si}$ as modeled by DFT-LDA with a potential in the form of eqn. (22), we obtain the following results: for the $\langle 211\rangle$ partial dislocation route along a $\{111\}$ glide plane, $L / b \approx 0.155, q \approx 0.647$ and $p \approx 0.747$; for the $\langle 110\rangle$ full dislocation slip route along a $\{111\}$ shuffle plane, $L / b \approx 0.0896, q \approx 0.675$ and $p \approx-0.881$ (this value of $p$ has the problem that $p^{2}>q$, 
which should not be allowed for the requirement that $\gamma_{\mathrm{us}}{ }^{\left(\mathrm{u}^{*}\right)}$ be positive, see next section). These values of $p$ were taken from the $\Delta_{\theta}{ }^{*}$ and $L$ values as given above. An alternative estimate of $p$, and hence $L$, can be obtained from the $\gamma_{\mathrm{us}}{ }^{(\mathrm{r})} / \gamma_{\mathrm{us}}{ }^{\langle\mathrm{u}\rangle}$ ratio, using eqn. (23). This leads to $p \approx 0.376$ (glide) and $p \approx-0.650$ (shuffle). From these $p$ values, and using the known $\Delta_{\theta}{ }^{*}$ values, we can also determine $L=\Delta_{\theta}{ }^{*} / p$ for the glide set as $0.0682 \mathrm{~nm}$ and for the shuffle set as $0.0466 \mathrm{~nm}$. Fortunately, we find that at such large values of $q$ as apply for $\mathrm{Si}$, the dislocation nucleation condition that we calculate by solving the integral equations has little dependence on $p$. Both estimates appear in Table 1. We use the latter estimates of $p$ in the dislocation nucleation calculations discussed subsequently.

\subsection{Relations of $\left(\Delta_{r}, \Delta_{\theta}\right)$ and $\Psi$ to $\left(\delta_{r}, \delta_{\theta}\right)$ and $\Phi$}

The upper case Greek letters $\left(\Delta_{r}, \Delta_{\theta}\right)$ denote the relative displacements of two adjacent atomic planes with initial normal separation $h$. Since the displacement discontinuities $\left(\delta_{r}, \delta_{\theta}\right)$ across a mathematical cut, introduced in our implementation of the Peierls concept at a crack tip, refer to a surface of zero thickness, we follow Rice [1] in expressing each $\Delta$ as the corresponding $\delta$ plus the additional displacement that would be acquired over distance $h$ between planes in the tensile and shear strain field in the adjoining elastic continuum corresponding to $\sigma$ and $\tau$. Evaluating those strains as for the linear elastic solid lying outside the cut, this gives additional displacements $(h \tau / \mu, h \sigma / c)$, where $\mu$ and $c$ are the shear and uniaxial strain tensile moduli respectively; $c=\lambda+2 \mu$ for an isotropic solid. Hence, we extract $\left(\delta_{r}, \delta_{\theta}\right)$ from $\left(\Delta_{r}, \Delta_{\theta}\right)$ by

$\delta_{r}=\Delta_{r}-h \tau\left(\Delta_{r}, \Delta_{\theta}\right) / \mu=\Delta_{r}-(h / \mu) \partial \psi\left(\Delta_{r}, \Delta_{\theta}\right) / \partial \Delta_{r}(24)$

$\delta_{\theta}=\Delta_{\theta}-h \sigma\left(\Delta_{r}, \Delta_{\theta}\right) / c=\Delta_{\theta}-(h / c) \partial \psi\left(\Delta_{r}, \Delta_{\theta}\right) / \partial \Delta_{\theta}(25)$

Since when $\left(\Delta_{r}, \Delta_{\theta}\right)$ approach zero,

$\tau\left(\Delta_{r}, \Delta_{\theta}\right) \rightarrow \mu \Delta_{r} / h \quad \sigma\left(\Delta_{r}, \Delta_{\theta}\right) \rightarrow c \Delta_{\theta} / h$

Equations (14) and (15) require for consistency that we understand here that $\mu / h=2 \pi^{2} \gamma_{\mathrm{us}}^{(\mathrm{u})} / b^{2}$ and that $c / h=2 \gamma_{\mathrm{s}} / L^{2}$. With those replacements, $h, \mu$ and $c$ do not appear explicitly in the relation between the stresses and displacements.

While $\tau\left(\Delta_{r}, \Delta_{\theta}\right)$ and $\sigma\left(\Delta_{r}, \Delta_{\theta}\right)$ are derivable from the potential $\Psi\left(\Delta_{r}, \Delta_{\theta}\right)$, the displacement discontinuities are associated with a potential $\Phi\left(\delta_{r}, \delta_{\theta}\right)$ for $\tau\left(\delta_{r}, \delta_{\theta}\right)$ and $\sigma\left(\delta_{r}, \delta_{\theta}\right)$ such that,

$\mathrm{d} \Phi\left(\delta_{r}, \delta_{\theta}\right)=\tau\left(\delta_{r}, \delta_{\theta}\right) \mathrm{d} \delta_{r}+\sigma\left(\delta_{r}, \delta_{\theta}\right) \mathrm{d} \delta_{\theta}$

(see eqns. (4) and (5)). Therefore, from the relations above between the $\Delta$ and $\delta$, from eqns. (24) and (25), and from eqn. (13), we derive that

$$
\begin{aligned}
\Phi\left(\delta_{r}, \delta_{\theta}\right)= & \Psi\left(\Delta_{r}, \Delta_{\theta}\right)-(1 / 2)(h / \mu) \tau^{2}\left(\Delta_{r}, \Delta_{\theta}\right) \\
& -(1 / 2)(h / c) \sigma^{2}\left(\Delta_{r}, \Delta_{\theta}\right)
\end{aligned}
$$

In formulating the integral eqns. (4) and (5) for the numerical solution we let $\left(\Delta_{r}, \Delta_{\theta}\right)$ serve as parametric variables in terms of which $\left(\delta_{r}, \delta_{\theta}\right), \Phi$ and $(\sigma, \tau)$ are determined.

\section{Results for dislocation nucleation}

Results for nucleation are now given in cases for which the emergent dislocation has both edge and screw components relative to the crack tip. Letting $\phi$ denote the angle between the Burgers vector and a line drawn normal to the crack front, in the slip plane, the vector thus has edge component $b \cos \phi$ and screw component $b \sin \phi$. Two cases when normal stress exists across the slip plane have been studied in detail. These are (1) mixed mode II and I loading on crack with a coplanar slip plane and emergent edge dislocation $(\theta=0$ and $\phi=0)$, and (2) pure mode I loading but for non-zero slip plane inclination $\theta$ and Burgers vector direction $\phi$ of the emergent dislocation.

\subsection{Mixed mode II and I loading, coincident crack and slip planes and emergent edge dislocation $(\theta=0$ and $\phi=0)$}

In the mixed mode II and I loading for coplanar crack and slip planes, the critical crack extension force $G_{\mathrm{d}}$ at dislocation nucleation depends on the phase angle $\psi$ of the mixed loading, where $\psi=\arctan \left(K_{\mathrm{II}} / K_{\mathrm{I}}\right)$. That is, $\psi=90^{\circ}$ for pure shear loading and $\psi=0^{\circ}$ for pure tension loading. Results for $G_{\mathrm{d}} / \gamma_{\mathrm{us}}{ }^{(\mathrm{u})}$ from numerical solution of the pair of coupled integral equations, based on the material parameters and slip systems of Table 1, are shown by the solid lines in Figs. $14(\alpha-\mathrm{Fe}), 15(\mathrm{Al}), 16(\mathrm{Ni}), 17\left(\mathrm{Ni}_{3} \mathrm{Al}\right), 18(\mathrm{Si}$, glide) and 19 ( $\mathrm{Si}$, shuffle). The flattening of the curves for small $\psi$, i.e. for conditions near pure mode I, corresponds in all cases to the Griffith decohesion loading $G_{\mathrm{d}}=2 \gamma_{\mathrm{s}}\left(\right.$ or $\left.G_{\mathrm{d}} / \gamma_{\mathrm{us}}^{(\mathrm{u})}=1 / q\right)$. That is also an upper limit to the load to nucleate a shear instability, in that the resistance to shearing becomes negligibly small as atomic planes are pulled apart. Our present modeling, which only detects the onset of an instability, does not tell us whether the subsequent instability itself should be regarded as more like a cleavage decohesion or more like a dislocation emission instability.

The solid-line exact numerical results in Figs. 14-19 are compared with a number of broken-line curves which represent different approximate estimates of the nucleation condition. These approximate estimates represent different attempts to interpret results of the shear-only model, that being the model which does not 


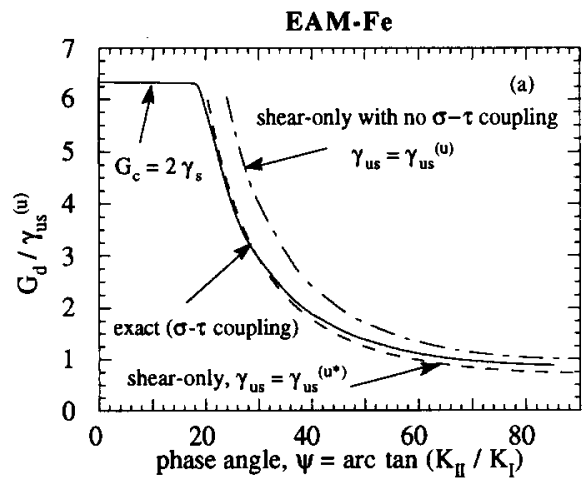

EAM-Fe

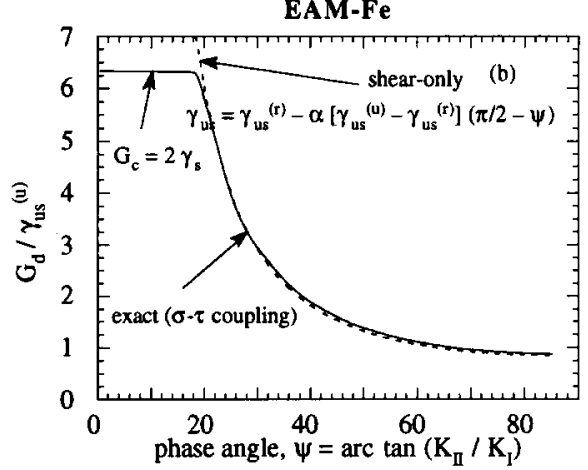

Fig. 14. The critical $G$ for emission of a full dislocation of edge type $(\phi=0)$ in (EAM) $\alpha-\mathrm{Fe} v s$, the loading phase angle $\left(\tan \psi=K_{\mathrm{II}} / K_{\mathrm{I}}\right)$ when the slip plane is copolanar with the crack plane $(\theta=0)$. The solid line is based on an exact numerical solution of eqns. (4)-(7) which takes into account coupling between tension and shear. The dashed line is based on a calculation for the shear-only model, for which the exact result is given by eqn. (1), and uses $\gamma_{\mathrm{us}}=\gamma_{\mathrm{us}}{ }^{(\mathrm{u})}$. The dash-dotted line is based on the same calculation but with $\gamma_{\mathrm{us}}=\gamma_{\mathrm{us}}{ }^{\left(\mathrm{u}^{*}\right)}$. Figure 14(b) is a comparison of the full tension-shear coupling model and the shear-only model but with $\gamma_{u s}(\psi)=\gamma_{u s}{ }^{(r)}-\alpha\left[\gamma_{u s}{ }^{(u)}-\gamma_{u s}{ }^{(r)}\right]$ $(\pi / 2-\psi)$, and $a=0.841$.

directly consider tension-shear coupling, and replaces the set of coupled equations (4)-(7) with the simpler equations (8) and (9) which consider shear response only. We recall that the exact solution of the shear-only model for the nucleation condition is known exactly and is given by eqn. (1). Thus, when expressed in terms of $G\left[G=(1-v)\left(K_{\mathrm{I}}^{2}+K_{\mathrm{II}}^{2}\right) / 2 \mu\right]$ using $K_{\mathrm{II}} / K_{\mathrm{I}}=\tan \psi$, the shear-only model gives

$$
G_{\mathrm{d}}=\gamma_{\mathrm{us}} / \sin ^{2} \psi
$$

(for small $\psi$, which corresponds to near mode I loading, this must be cut off at the Griffith tensile decohesion loading $G_{\mathrm{d}}=2 \gamma_{\mathrm{s}}$ ).

The uppermost broken-line curves in parts (a) of Figs. 14-19 show the result of eqn. (29) when we identify $\gamma_{u s}$ as the unrelaxed value $\gamma_{u s}{ }^{(u)}$. This clearly overestimates the exact (solid-line) results. One does a slightly better job by identifying $\gamma_{u s}$ in eqn. (29) as the
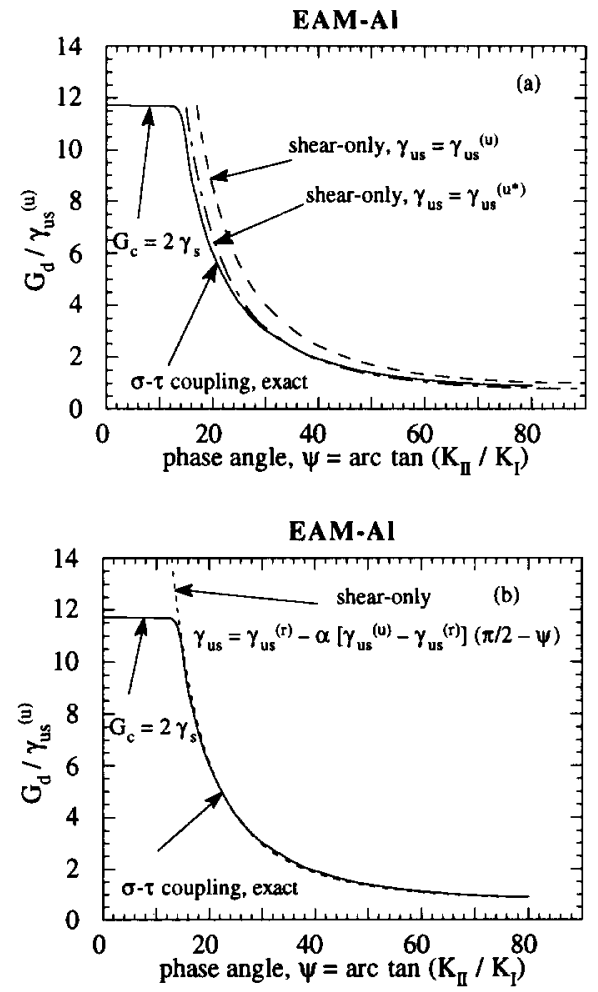

Fig. 15. The critical $G$ for emission of a Shockley dislocation of edge character in EAM $\mathrm{Al} v s$. the loading phase angle $\left(\tan \psi=K_{\mathrm{II}} / K_{\mathrm{l}}\right)$ when the slip plane is coplanar with the crack plane $(\theta=0)$. The solid line is based on an exact numerical solution of the tension-shear coupling model. The dashed line is based on a calculation for the shear-only model, for which the exact result is given by eqn. (1), and uses $\gamma_{u s}=\gamma_{u s}{ }^{(u)}$. The dashdotted line is based on the same calculation but with $\gamma_{\mathrm{us}}=\gamma_{\mathrm{us}}{ }^{\left(\mathrm{u}^{*}\right)}$. Figure $15(\mathrm{~b})$ is a comparison of the full tension-shear coupling model and the shear-only model but with $\gamma_{\mathrm{us}}(\psi)=\gamma_{\mathrm{us}}(\mathrm{r})-$ $\alpha\left[\gamma_{\mathrm{us}}{ }^{(\mathrm{u})}-\gamma_{\mathrm{us}}{ }^{(\mathrm{r})}\right](\pi / 2-\psi)$, and $\alpha=1.145$.

relaxed value $\gamma_{u s}^{(r)}$, but that too overestimates the exact results except at large $\psi$, near $90^{\circ}$ and corresponding to conditions near pure mode II loading. Rice et al. [12] found that results from the shear-only model, eqn. (29), could be made to agree approximately with the exact results for EAM $\alpha-\mathrm{Fe}, \mathrm{Al}$ and $\mathrm{Ni}$ if $\gamma_{\mathrm{us}}$ was identified as a quantity $\gamma_{\mathrm{us}}{ }^{\left(\mathrm{u}^{*}\right)}$ which is a modified type of unrelaxed unstable stacking energy. The results with this choice are shown as the lower broken-line curve in parts (a) of Figs. 14-19, and the agreement is also seen to be good for the EAM Ni $\mathrm{Al}_{3} \mathrm{Aase}$, although not for either of the DFT-LDA Si cases.

The new quantity is defined by $\gamma_{\text {us }}{ }^{\left({ }^{*}\right)}=\int \tau\left(\Delta_{r}, \Delta_{\theta}{ }^{*}\right) \mathrm{d} \Delta_{r}$ from $\Delta_{r}=0$ to $b / 2$, where $\Delta_{\theta}{ }^{*}$ is as previously defined. For comparison, $\gamma_{\mathrm{us}}^{(\mathrm{u})}=\int \tau\left(\Delta_{r}, 0\right) \mathrm{d} \Delta_{r}$ from $\Delta_{r}=0$ to $b / 2$, and $\gamma_{\mathrm{us}}{ }^{\mathrm{r}}{ }^{\mathrm{r})}=\int \tau\left(\Delta_{r}, \Delta_{\theta}{ }^{\mathrm{r}}\right) \mathrm{d} \Delta_{r}$ from $\Delta_{r}=0$ to $b / 2$ where $\Delta_{\theta}{ }^{r}$ is the function of $\Delta_{r}$ which makes $\sigma=0$ at each $\Delta_{r}$ In terms of the parametrized combined shear and tension constitutive law, 


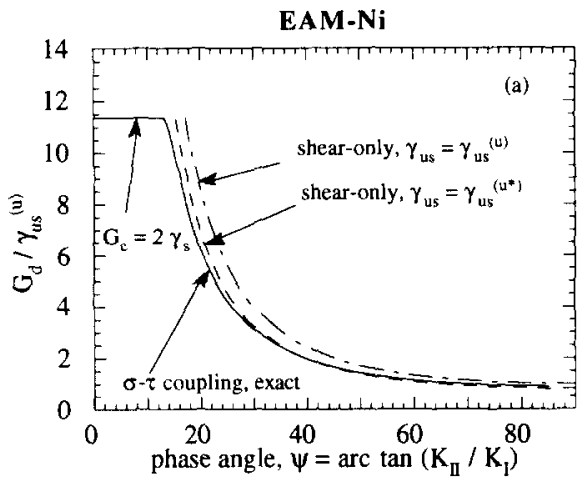

EAM-Ni

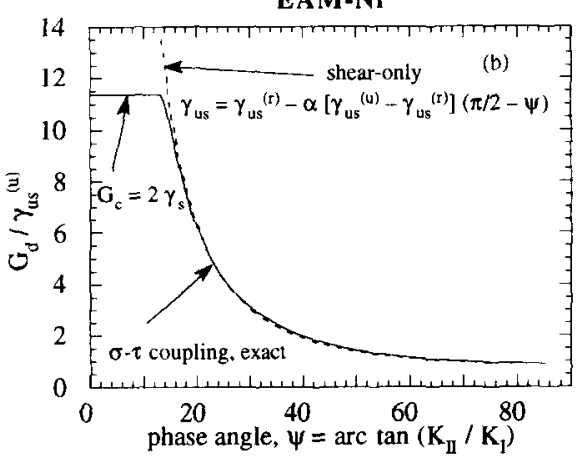

Fig. 16. The critical $G$ for emission of a Shockley dislocation of edge character in EAM $\mathrm{Ni}$ vs. the loading phase angle $\left(\tan \psi=K_{\mathrm{fl}} / K_{\mathrm{I}}\right)$ when the slip plane is coplanar with the crack plane $(\theta=0)$. The solid line is based on an exact numerical solution of the tension-shear coupling model. The dashed line is based on a calculation for the shear-only model, for which the exact result is given by eqn. (1), and uses $\gamma_{u s}=\gamma_{u s}{ }^{(u)}$. The dashdotted line is based on the same calculation but with $\gamma_{\mathrm{us}}=\gamma_{\mathrm{us}}{ }^{\left(\mathrm{u}^{*}\right)}$. Figure $16(b)$ is a comparison of the full tension-shear coupling model and the shear-only model but with $\gamma_{\mathrm{us}}(\psi)=\gamma_{\mathrm{us}}(\mathrm{r})-$ $\alpha\left[\gamma_{\mathrm{us}}{ }^{(\mathrm{u})}-\gamma_{\mathrm{us}}{ }^{(\mathrm{r})}\right](\pi / 2-\psi)$, and $\alpha=1.323$.

$\gamma_{\mathrm{us}}{ }^{\left(\mathrm{u}^{*}\right)}=\gamma_{\mathrm{us}}{ }^{(\mathrm{u})}\left(\frac{q-p^{2}}{q(1-p)}\right) \exp (-p)$

The $\gamma_{\mathrm{us}}{ }^{\left\langle u^{*}\right\rangle}, \gamma_{\mathrm{us}}{ }^{\langle\mathrm{u}\rangle}$ and $\gamma_{\mathrm{us}}{ }^{(\mathrm{r})}$ values for $\mathrm{Fe}, \mathrm{Al}, \mathrm{Ni}$ and $\mathrm{Ni}_{3} \mathrm{Al}$ are listed in Table 2 as calculated from EAM and in Table 3 from the parametrized combined tension-shear constitutive laws, which also gives estimates for the Si glide and shuffle cases. It is interesting to note that the ratio $\gamma_{\mathrm{us}}{ }^{(\mathrm{u})} / \gamma_{\mathrm{us}}{ }^{(\mathrm{r})}$ is roughly the same as $\gamma_{\mathrm{us}}^{(\mathrm{r})} / \gamma_{\mathrm{us}}{ }^{\left(\mathrm{us}^{*}\right)}$ for EAM Fe, Al, Ni and $\mathrm{Ni}_{3} \mathrm{Al}$.

A somewhat better approximation is, again, to use the shear-only model of eqn. (29) but to let the unstable stacking energy $\gamma_{\mathrm{us}}$ which enters it be dependent on the ratio of tensile to shear loading. We do this by writing

$\gamma_{\mathrm{us}}(\psi)=\gamma_{\mathrm{us}}{ }^{(\mathrm{r})}-\alpha\left[\gamma_{\mathrm{us}}{ }^{(\mathrm{u})}-\gamma_{\mathrm{us}}{ }^{(\mathrm{r})}\right](\pi / 2-\psi)$

where $\psi$ is, as above, the phase angle of the mixed loading in radians, and $\alpha$ is a reduction coefficient,
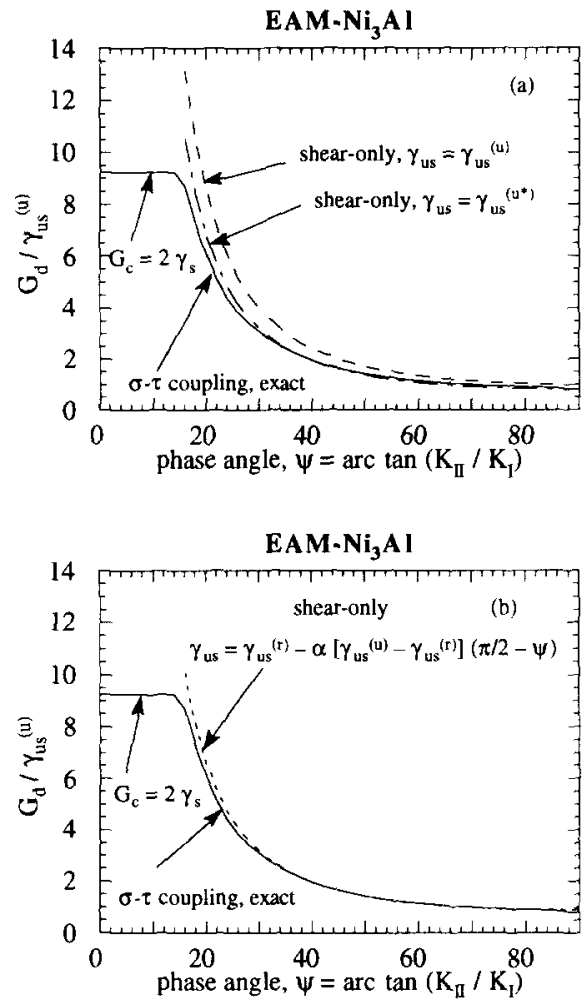

Fig. 17. The critical $G$ for emission of a Shockley partial dislocation of edge character in $\mathrm{EAM} \mathrm{Ni} \mathrm{Al}_{3} \mathrm{Al}$ s. the loading phase angle $\left(\tan \psi=K_{\mathrm{II}} / K_{\mathrm{I}}\right)$ when the slip plane is coplanar with the crack plane $(\theta=0)$. The solid line is based on an exact numerical solution of the tension-shear coupling model. The dashed line is based on a calculation for the shear-only model, for which the exact result is given by eqn. (1), and uses $\gamma_{u s}=\gamma_{u s}\langle u\rangle$. The dashdotted line is based on the same calculation but with $\gamma_{u s}=\gamma_{u s}\left(u^{*}\right)$. Figure $17(b)$ is a comparison of the full tension-shear coupling model and the shear-only model but with $\gamma_{\text {us }}(\psi)=\gamma_{u s}{ }^{(r)}-$ $\alpha\left[\gamma_{\mathrm{us}}{ }^{(\mathrm{u})}-\gamma_{\mathrm{us}}{ }^{(\mathrm{r})}\right](\pi / 2-\psi)$, and $\alpha=0.969$.

determined by fitting to the exact tension-shear coupled results. This procedure then leads to $G_{\mathrm{d}}=\gamma_{\mathrm{us}}(\psi) / \sin ^{2} \psi$, which result is shown by the broken-line curves in parts (b) of Figs. 14-19. We do not know $\alpha$ a priori, but it is interesting that it turns out to be around unity for all the metals we have studied. In particular, $\alpha$ has the values $1.323,1.145,0.969$ and 0.841 for $\mathrm{Ni}, \mathrm{Al}, \mathrm{Ni}_{3} \mathrm{Al}$ and $\mathrm{Fe}$ respectively, and the exact results for those cases are described well by this approximation.

For Si, Figs. 18 and 19, the tension-reduced $\gamma_{u s}(\psi)$ also gives a better fit to the tension-shear coupled results than does $\gamma_{\mathrm{us}}{ }^{\left({ }^{*}\right)}$, but the $\alpha$ values are in a very different range. The value of $\alpha$ for the glide set is determined to be 7.249. For the shuffle set it is interesting to note that $p$ is negative. The tension-reduced $\gamma_{\mathrm{us}}(\psi)$ gives a somewhat valid description. The value of $\alpha$ for the shuffle set is determined to be -2.234 ; note that the $\alpha$ coefficient is also negative. 

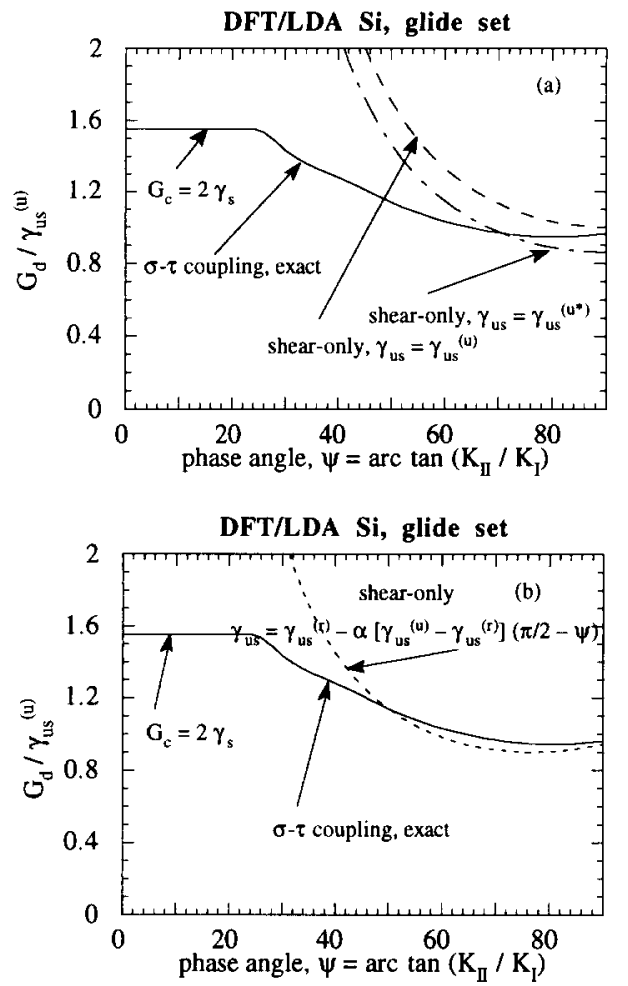

Fig. 18. The critical $G$ for emission of a Shockley partial dislocation of edge character on the DFT-LDA Si glide set $v s$. the loading phase angle $\left(\tan \psi=K_{11} / K_{1}\right)$ when the slip plane is coplanar with the crack plane $(\theta=0)$. The solid line is based on an exact numerical solution of the tension-shear coupling model. The dashed line is based on a calculation for the shear-only model, for which the exact result is given by eqn. (1), and uses $\gamma_{u s}=\gamma_{u s}{ }^{(u)}$. The dash-dotted line is based on the same calculation but with $\gamma_{\mathrm{us}}=\gamma_{\mathrm{us}}{ }^{\left(\mathrm{u}^{*}\right)}$. Figure $18(\mathrm{~b})$ is a comparison of the full tension-shear coupling model and the shear-only model but with $\gamma_{\mathrm{us}}(\psi)=\gamma_{\mathrm{us}}{ }^{(\mathrm{r})}-\alpha\left[\gamma_{\mathrm{us}}{ }^{(\mathrm{u})}-\gamma_{\mathrm{us}}{ }^{(\mathrm{r})}\right](\pi / 2-\psi)$, and $\alpha=7.249$.

\subsection{General dependence on tension-shear parameters over a wide range for mixed mode II and I loading and for $\theta=0$ and $\phi=0$}

The materials considered in this study show a wide range of ratio of unstable stacking energy to twice the surface energy (i.e. q). As a general rule-of-thumb, the materials studied here suggest that for f.c.c. metals including $\mathrm{Ni}_{3} \mathrm{Al}, q \approx 0.1$; for the one b.c.c. metal $q \approx 0.2$, and for the semiconductor $\mathrm{Si}$, both glide and shuffle cases, $q \approx 0.6$. If we assume that all materials possess a $q$ value that falls into this kind of range, it is possible to assess quickly the critical loading for dislocation nucleation from a crack tip, taking into account the tension-shear coupling. We generate diagrams of critical $K_{\mathrm{II}}$ scaled by $\left[2 \mu \gamma_{\mathrm{us}}{ }^{(\mathrm{r})} /(1-v)\right]^{1 / 2} v s$. the phase angle $\psi=\arctan \left(K_{\mathrm{II}} / K_{\mathrm{I}}\right)$, in order to show how it depends on $q$, and on a finer scale, the dilation parameter $p$. The results are shown in Figs. 20-25. An interesting feature from the studies is that for high $q$ values, the critical
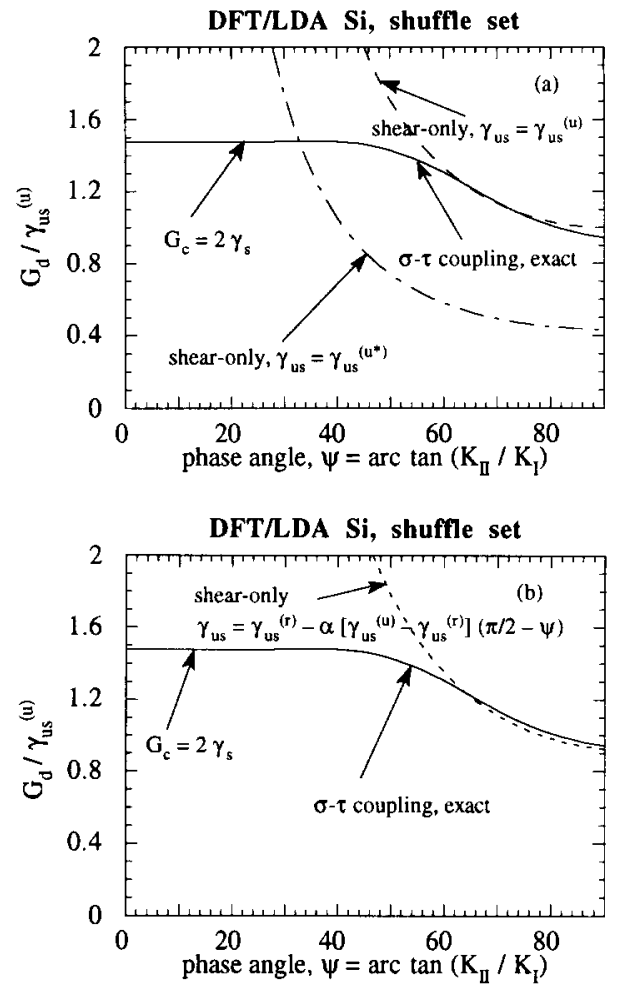

Fig. 19. The critical $G$ for emission of a full dislocation of edge character on the DFT-LDA Si shuffle set $v s$. the loading phase angle $\left(\tan \psi=K_{\mathrm{II}} / K_{\mathrm{I}}\right)$ when the slip plane is coplanar with the crack plane $(\theta=0)$. The solid line is based on an exact numerical solution of the tension-shear coupling model. The dashed line is based on a calculation for the shear-only model, for which the exact result is given by eqn. (1), and uses $\gamma_{u s}=\gamma_{u s}{ }^{(u)}$. The dashdotted line is based on the same calculation but with $\gamma_{\mathrm{us}}=\gamma_{\mathrm{us}}{ }^{\left(\mathrm{u}^{*}\right)}$. Figure 19(b) is a comparison of the full tension-shear coupling model and the shear-only model but with $\gamma_{\mathrm{us}}(\psi)=\gamma_{\mathrm{us}}{ }_{\mathrm{r}}{ }_{-}$ $\alpha\left[\gamma_{\mathrm{us}}{ }^{(\mathrm{u})}-\gamma_{\mathrm{us}}{ }^{(\mathrm{r})}\right](\pi / 2-\psi)$, and $\alpha=-2.234$.

condition is insensitive to the value of the dilation parameter $p$.

For convenience, a parameter $z$ is introduced, defined as $z=\gamma_{\mathrm{us}}{ }_{\mathrm{r}}^{(\mathrm{r})} / 2 \gamma_{\mathrm{s}}$, which can be found from $q$ and $p$,

$z=q-\left(\frac{1-q}{1-p}\right)[\exp (-p)-1+p]$

Each of Figs. $20-25$ is characterized by a different value of $z(z=0.1,0.2,0.3,0.4,0.6$ and 0.8$)$. Different curves in the same figure are for various $p$ values $(p=0.1,0.2,0.3,0.4,0.6$ and 0.8$)$. The maximum absolute value of $p$ may not be larger than $\sqrt{q}$. This is evident from eqn. (29), because we require that the sliding displacement at $b / 2$ to be higher in energy than at zero sliding displacement, with the opening separation fixed at $\Delta_{\theta}{ }^{*}$, or in short that the quantity $\gamma_{u s}{ }^{\left(u^{*}\right)}$ be positive. 


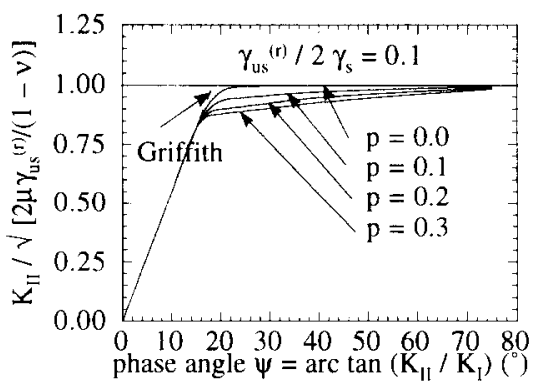

Fig. 20. The family of curves for $\gamma_{\mathrm{us}}^{(\mathrm{r})} / 2 \gamma_{\mathrm{s}}=0.1$ showing how the critical mode II loading dislocation nucleation in the coplanar slip and crack planes case depends on the mode II-mode I loading phase angle and on various $p$ values.

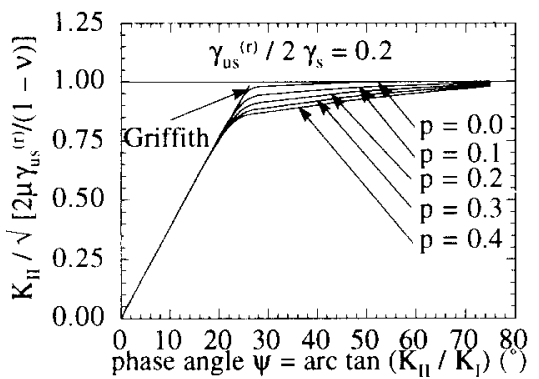

Fig. 21. The family of curves for $\gamma_{\mathrm{us}}^{(\mathrm{r})} / 2 \gamma_{\mathrm{s}}=0.2$ showing how the critical mode II loading dislocation nucleation in the coplanar slip and crack planes case depends on the mode II-mode I loading phase angle and on various $p$ values.

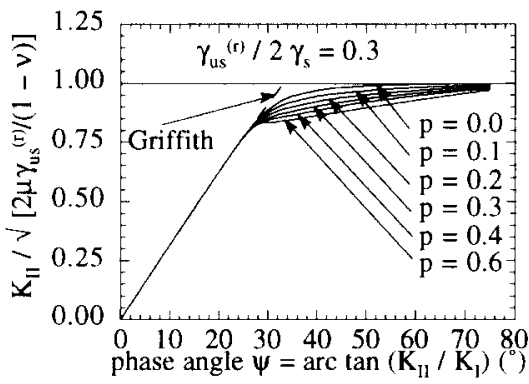

Fig. 22. The family of curves for $\gamma_{\text {us }}^{(r)} / 2 \gamma_{s}=0.3$ showing how the critical mode II loading dislocation nucleation in the coplanar slip and crack planes case depends on the mode II-mode I loading phase angle and on various $p$ values.

For small phase angles, under mixed mode I and II, the approach of the combined tension-shear model becomes coincident with the Griffith decohesion condition $G=2 \gamma_{\mathrm{s}}$, as discussed in connection with the flat portions of the solid line curves in Figs. 14-19. Thus, at small $\psi, G_{\mathrm{d}} \approx 2 \gamma_{\mathrm{s}}$ and

$$
K_{\mathrm{II}} /\left[2 \mu \gamma_{\mathrm{us}}{ }^{(\mathrm{r})} /(1-v)\right]^{1 / 2} \approx(1 / \sqrt{z}) \sin \psi
$$

For large $\psi$, near pure mode II loading conditions, there is negligible tensile stress on the slip plane and

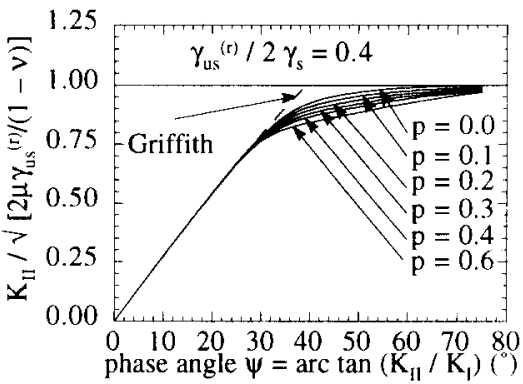

Fig. 23. The family of curves for $\gamma_{u s}{ }^{(r)} / 2 \gamma_{s}=0.4$ showing how the critical mode II loading dislocation nucleation in the coplanar slip and crack planes case depends on the mode II-mode I loading phase angle and on various $p$ values.

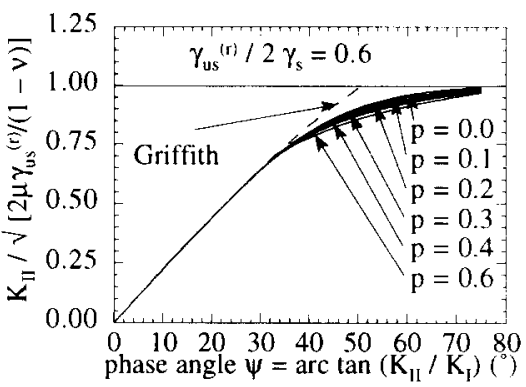

Fig. 24. The family of curves for $\gamma_{\mathrm{us}}{ }^{i t} / 2 \gamma_{\mathrm{s}}=0.6$ showing how the critical mode II loading dislocation nucleation in the coplanar slip and crack planes case depends on the mode II-mode I loading phase angle and on various $p$ values.

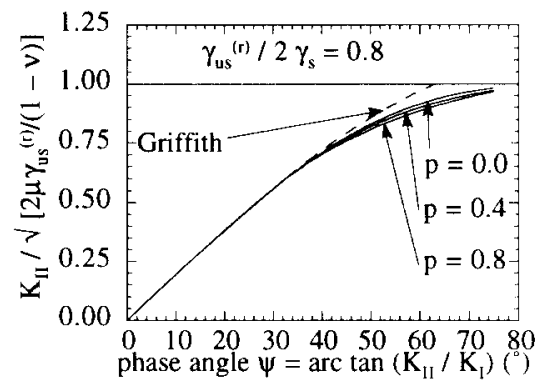

Fig. 25. The family of curves for $\gamma_{\mathrm{us}}^{(\mathbf{r})} / 2 \gamma_{\mathrm{s}}=0.8$ showing how the critical mode II loading dislocation nucleation in the coplanar slip and crack planes case depends on the mode II-mode I loading phase angle and on various $p$ values.

the nucleation by the shear-only result of eqn. (1) with $\gamma_{\mathrm{us}}=\gamma_{\mathrm{us}}{ }^{(\mathrm{r})}$. Thus we expect, for $\psi$ near $\pi / 2$, that

$K_{\mathrm{II}} /\left[2 \mu \gamma_{\mathrm{us}}^{(\mathrm{r})} /(1-v)\right]^{1 / 2} \approx 1$

which is indicated by a horizontal line of value unity in Figs. 20-25.

The reduction in the critical shear loading $K_{\mathrm{II}}$ for nucleation because of tension-shear coupling is shown by the fact that all the critical $K_{\text {II }} v s$. $\psi$ curves are below the horizontal line of unity in the series of figures. 
4.3. Mode I loading, with inclined slip planes and mixed edge-screw character of emergent dislocation $(\theta \neq 0$ and $\phi \neq 0$ )

For the more general case involving screw components $\phi \neq 0$, the incipient profile is of the type $\left\{\delta_{r}(r), \delta_{\theta}(r), \delta_{z}(r)\right\}$ in the slip plane making an angle $\theta$ with the crack plane, as in Fig. 1. Following Rice [1], we apply the constrained slip path approximation here, treating the slip as constrained to be along the direction $\boldsymbol{s}$ (the same as $\boldsymbol{b}$ ) that makes an angle $\phi$ with the $\boldsymbol{r}$ axis in the slip plane, $\delta_{\alpha}(r)=\left[\delta_{s}(r) \cos \phi, \delta_{\theta}(r), \delta_{s}(r) \sin \phi\right]$. The corresponding shear and normal stresses are $\tau=\sigma_{\theta r} \cos \phi+\sigma_{\theta z} \sin \phi, \sigma=\sigma_{\theta \theta}$. In place of eqns. (4) and (5) for the tension-shear coupling model, we have the following integral equations:

$$
\begin{aligned}
\tau\left[\delta_{s}(r), \delta_{\theta}(r)\right]= & \frac{K_{\tau}^{\text {eff }}}{(2 \pi r)^{1 / 2}}-\int_{0}^{\infty} \bar{g}_{11}(r, s ; \theta, \phi) \frac{\mathrm{d} \delta_{s}(s)}{\mathrm{d} s} \mathrm{~d} s \\
& -\int_{0}^{\infty} \bar{g}_{12}(r, s ; \theta, \phi) \frac{\mathrm{d} \delta_{\theta}(s)}{\mathrm{d} s} \mathrm{~d} s \\
\sigma\left[\delta_{s}(r), \delta_{\theta}(r)\right]= & \frac{K_{\sigma}^{\text {eff }}}{(2 \pi r)^{1 / 2}}-\int_{0}^{\infty} \bar{g}_{21}(r, s ; \theta, \phi) \frac{\mathrm{d} \delta_{s}(s)}{\mathrm{d} s} \mathrm{~d} s \\
& -\int_{0}^{\infty} \bar{g}_{22}(r, s ; \theta, \phi) \frac{\mathrm{d} \delta_{\theta}(s)}{\mathrm{d} s} \mathrm{~d} s
\end{aligned}
$$

where $\quad K_{\tau}^{\text {eff }}=(2 \pi r)^{1 / 2}\left[\cos \phi \sigma_{\theta r}{ }^{0}(r, \theta)+\sin \phi \sigma_{\theta z}{ }^{0}(r, \theta)\right]$, and $K_{\sigma}{ }^{\text {eff }}=(2 \pi r)^{1 / 2} \sigma_{\theta \theta}{ }^{0}(r, \theta)$ are the effective stress intensity factors for the singular stresses $\sigma_{\theta r}{ }^{0}, \sigma_{\theta z}{ }^{0}$ and $\sigma_{\theta \theta}{ }^{0}$ at the crack tip under external loading before the emergence of the incipient profile. Under pure mode I loading they are given by $K_{\tau}^{\text {eff }}=K_{\mathrm{I}} \cos \phi$ $\sin (\theta / 2) \cos ^{2}(\theta / 2)$ and $K_{\sigma}^{\text {eff }}=K_{\mathrm{I}} \cos ^{3}(\theta / 2)$.

The terms $\bar{g}_{11}, \bar{g}_{12}, \bar{g}_{21}$ and $\bar{g}_{22}$ are the stress functions of a straight dislocation with mixed edge and screw components near a crack tip. All of these terms and functions can be obtained using the linear isotropic or anisotropic elasticity formulation. For details of these functions, see Sun and Berlitz [13]. The terms $\tau\left[\delta_{s}(r), \delta_{\theta}(r)\right]$ and $\sigma\left[\delta_{s}(r), \delta_{\theta}(r)\right]$ are the same shear and tension stresses across the slip plane as before, expressed in eqns. (6) and (7), and the same analytical formulae as described in Section 3 are used to represent these functions.

In the shear only model, the equation to be solved becomes, in place of eqn. (8),

$$
\tau\left[\delta_{s}(r)\right]=\frac{K_{\tau}^{\text {eff }}}{(2 \pi r)^{1 / 2}}-\int_{0}^{\infty} \bar{g}_{11}(r, s ; \theta, \phi) \frac{\mathrm{d} \delta_{s}(s)}{\mathrm{d} s} \mathrm{~d} s
$$

The function $\tau\left[\delta_{s}(r)\right]$ is expressed as in eqn. (9). Rice [1] found the exact nucleation solution to the shear-only model when $\theta=0$. The result is

$K_{\tau}^{\mathrm{eff}}=\left\{2 \mu \gamma_{\text {us }}\left[\cos ^{2} \phi+(1-v) \sin ^{2} \phi\right] /(1-v)\right\}^{1 / 2}$

which generalizes eqn. (1) and reduces to it when $\phi=0$. He proposed, in fact, that eqn. (38) be used as an approximation for situations with $\theta \neq 0$, like for pure mode I loading considered in this subsection.

We may therefore compare the exact numerical results from solutions to the tension-shear coupled integral equations, eqns. (35) and (36), with two types of simpler expressions. The first, and simplest, is based on using the effective shear stress intensity factor concept, and amounts to using eqn. (38) with a special choice of the $\gamma_{u s}$ term which enters it to account approximately for tension-shear coupling. The second is based on exact numerical solution of the shear-only integral equation, eqn. (37), with the $\tau v s$. $\delta_{s}$ function taken as that consistent with a Frenkel-Peierls sinusoid of form $\tau=\left(\pi \gamma_{\text {us }} / b\right) \sin \left(2 \pi \Delta_{s} / b\right)$. Here too the $\gamma_{\text {us }}$ term may be chosen to account approximately for tension-shear coupling.

One such choice would be simply to take $\gamma_{\mathrm{us}}$ as $\gamma_{\mathrm{us}}{ }^{(\mathrm{r})}$. However, what we have learned previously in study of the case $\theta=0$ suggests that we should take $\gamma_{u s}$ as the tension-softened $\gamma_{u s}(\psi)$ of eqn. (31), where now the logical interpretation of the loading phase angle $\psi$ is as $\psi=\arctan \left(K_{\tau}^{\text {eff }} / K_{o}^{\text {eff }}\right)$. For pure mode I loading this reduces to $\psi=\arctan [\cos \phi \tan (\theta / 2)]$, and just to $\psi=\theta / 2$ when the emergent dislocation is of edge character so that $\phi=0$. Of course, the prediction based on this choice of $\gamma_{u s}(\psi)$ should be cut off by a Griffith decohesion threshold at sufficiently small $\psi$, as in Figs. 14-19.

Table 4 summarizes results for the nucleation condition, in the form $G_{\mathrm{d}} / \gamma_{\mathrm{us}}{ }^{(\mathrm{r})}$, for pure mode I loading and for various $\theta$ and $\phi$ values, for the materials and slip systems listed in Table 1. The column labeled "full $\sigma-\tau$ coupling" gives the exact results found by numerical solution of the system of coupled integral equations in eqns. (35) and (36). The column labeled "based on $K_{r}$ eff" is the result of simply using eqn. (38), which is reexpressed in terms of $G$, for the present pure mode I loading, to give

$$
G_{\mathrm{d}}=8 \gamma_{\mathrm{us}}\left[1+(1-v) \tan ^{2} \phi\right] /\left[(1+\cos \theta) \sin ^{2} \theta\right]
$$

For that column of the table we have identified $\gamma_{\text {us }}$ as $\gamma_{\mathrm{us}}{ }^{\langle\mathrm{r}\rangle}$. The column labeled "shear-only" contains two sets of numbers, both based on the numerical solution of the shear-only integral equation, eqn. (37). The first set corresponds to choosing $\gamma_{\mathrm{us}}$ as $\gamma_{\mathrm{us}}{ }^{(\mathrm{r})}$. The second set, in square brackets, corresponds to choosing $\gamma_{\text {us }}$ as $\gamma_{\text {us }}(\psi)$ of eqn. (31), basing $\psi$ on the effective stress intensities as above and using the values of $\alpha$ in eqn. (31) quoted earlier. 
TABLE 4. Comparison of the critical $G_{\mathrm{d}}$ for dislocation emission on inclined slip planes under mode I loading

\begin{tabular}{|c|c|c|c|c|}
\hline $\begin{array}{l}\theta, \phi \\
(\mathrm{deg})\end{array}$ & $\begin{array}{l}G_{\mathrm{d}} / \gamma_{\mathrm{us}}{ }^{\mathrm{r})} \\
\text { (based on } K_{\tau}^{\mathrm{eff}} \text { ) }\end{array}$ & $\begin{array}{l}G_{\mathrm{d}} / \gamma_{\mathrm{us}}\{\mathrm{n} \\
\text { (shear-only) } \\
{\left[\text { with } \gamma_{\mathrm{us}}(\psi)\right]}\end{array}$ & $\begin{array}{l}G_{\mathrm{d}} / \gamma_{\mathrm{us}}{ }^{i \mathrm{r}} \\
\text { (full } \sigma^{-} \tau \\
\text { coupling) }\end{array}$ & $\begin{array}{l}\text { Reduction }(\%) \\
\left(K_{\text {II }}^{\text {eff }} \text { to full }\right. \\
\sigma-\tau \text { coupling) }\end{array}$ \\
\hline \multicolumn{5}{|c|}{$\mathrm{EAM} \mathrm{Fe}$ (b.c.c.), full $(v=0.324, p=0.214, q=0.158, L / b=0.197)$} \\
\hline 45,0 & 9.37 & $8.34[6.94]$ & 6.65 & 29.0 \\
\hline $45,35.3$ & 12.5 & $11.4[9.35]$ & 9.18 & 26.8 \\
\hline 90,0 & 8.00 & $5.92[5.26]$ & 5.40 & 32.5 \\
\hline $90,35.3$ & 10.7 & $8.20[7.17]$ & 7.53 & 29.6 \\
\hline $90,54.7$ & 18.8 & $15.1[12.8]$ & 13.4 & 28.9 \\
\hline \multicolumn{5}{|c|}{ EAM Al (f.c.c. $)$ partial $(v=0.345, p=0.140, q=0.0854, L / b=0.279)$} \\
\hline $35.3,0$ & 13.2 & $12.2 \quad[9.84]$ & 9.72 & 26.4 \\
\hline $54.7,0$ & 7.61 & $6.43[5.35]$ & 5.58 & 26.7 \\
\hline $54.7,60$ & 22.6 & $20.6 \quad[16.4]$ & 16.0 & 28.9 \\
\hline $70.5,0$ & 6.75 & $5.29[4.51]$ & 4.81 & 28.7 \\
\hline 90,0 & 8.00 & $5.92[5.21]$ & 5.47 & 31.6 \\
\hline 90,30 & 9.75 & $7.40 \quad[6.43]$ & 6.87 & 29.6 \\
\hline \multicolumn{5}{|c|}{ EAM Ni (f.c.c.), partial $(v=0.281, p=0.132, q=0.0879, L / b=0.271)$} \\
\hline $35.3,0$ & 13.2 & $12.2[9.95]$ & 9.82 & 25.6 \\
\hline $54.7,0$ & 7.61 & $6.43[5.40]$ & 5.64 & 25.9 \\
\hline $54.7,60$ & 24.0 & $22.0[17.7]$ & 17.4 & 27.5 \\
\hline $70.5,0$ & 6.75 & $5.29[4.55]$ & 4.85 & 28.2 \\
\hline 90,0 & 8.00 & $5.92[5.24]$ & 5.53 & 30.9 \\
\hline 90,30 & 9.92 & $7.54[6.59]$ & 7.03 & 29.1 \\
\hline \multicolumn{5}{|c|}{$\mathrm{EAM} \mathrm{Ni}_{3} \mathrm{Al}\left(\mathrm{L}_{1}\right)$, partial $(v=0.263, p=0.150, q=0.108, L / b=0.256)$} \\
\hline $35.3,0$ & 13.2 & $12.2[10.5]$ & 9.70 & 26.6 \\
\hline $54.7,0$ & 7.61 & $6.43[5.64]$ & 5.60 & 26.4 \\
\hline $54.7,60$ & 24.4 & $22.8[19.4]$ & 18.5 & 24.2 \\
\hline $70.5,0$ & 6.75 & $5.29[4.72]$ & 4.83 & 28.5 \\
\hline 90,0 & 8.00 & $5.92[5.40]$ & 5.54 & 30.7 \\
\hline 90,30 & 9.97 & $7.58[6.85]$ & 7.05 & 29.3 \\
\hline \multicolumn{5}{|c|}{ DFT-LDA Si (diamond), glide, partial $(v=0.218, p=0.376, q=0.647, L / b=0.308)$} \\
\hline $35.3,0$ & 13.2 & $12.2 \quad[5.74]$ & $1.99(\mathrm{C})$ & \\
\hline $54.7,0$ & 7.61 & $6.43[3.48]$ & $2.57(C)$ & \\
\hline $54.7,60$ & 25.5 & $23.3[10.4]$ & $3.03(\mathrm{C})$ & \\
\hline $70.5,0$ & 6.75 & $5.29[3.17]$ & 3.18 & 53.0 \\
\hline $70.5,60$ & 22.6 & $19.7[9.52]$ & $4.66(C)$ & \\
\hline 90,0 & 8.00 & $5.92[3.97]$ & 4.82 & 39.8 \\
\hline 90,30 & 10.1 & $7.68[4.92]$ & 5.70 & 43.5 \\
\hline \multicolumn{5}{|c|}{ DFT-LDA Si (diamond), shuffle, full ( $v=0.218, p=-0.650, q=0.675, L / b=0.121)$} \\
\hline $35.3,30$ & 16.7 & $15.6[19.4]$ & $1.98(\mathrm{C})$ & \\
\hline $54.7,0$ & 7.61 & $6.43[7.75]$ & $2.57(\mathrm{C})$ & \\
\hline $54.7,30$ & 9.59 & $8.30[10.1]$ & $2.68(\mathrm{C})$ & \\
\hline $70.5,30$ & 8.51 & $6.90 \quad[8.22]$ & $3.80(\mathrm{C})$ & \\
\hline 90,0 & 8.00 & $5.92 \quad[6.79]$ & 6.10 & 23.8 \\
\hline
\end{tabular}

$G_{\mathrm{d}}$ values in the first column are calculated based on the $K_{\mathrm{t}}^{\mathrm{eff}}$ concept in which $\gamma_{\mathrm{us}}$ is identified with $\gamma_{\mathrm{us}}$ (r)

$G_{\mathrm{d}}$ values in the second column are calculated from the numerical solution to eqns. $(8)$ and $(9)$, or to eqns. $(37)$ and $(9)$ when $\phi \neq 0$, which only take slip into account. Again, $\gamma_{\mathrm{us}}$ in this procedure is identified with $\gamma_{\mathrm{us}}{ }^{(\mathrm{r})}$. The bracketed value is what is predicted when $\gamma_{\mathrm{us}}$ is taken as $\gamma_{\mathrm{us}}(\psi)=\gamma_{\mathrm{us}}{ }_{\mathrm{i}} \mathrm{r}-\alpha\left[\gamma_{\mathrm{us}}{ }^{(\mathrm{u})}-\gamma_{\mathrm{us}}{ }^{(\mathrm{r})}\right](\pi / 2-\psi)$ in order to estimate the normal stress effect; $\psi=\arctan \left(K_{\tau}{ }^{\text {eff }} / K_{\sigma}{ }^{\text {eff }}\right)=\arctan (\cos \phi$ $\tan \theta / 2)$ for mode I loading.

$G_{\mathrm{d}}$ values in the third column take into account the full coupling between tension and shear and are based on the numerical solution to eqns. (4)-(7), or of eqns. (35), (36), (6) and (7) when $\phi \neq 0$.

The sign $(C)$ indicates that the instability occurs along the branch of results analogous to the flat portions of the solid curves in Figs. 18 and 19 , and may correspond to Griffith cleavage decohesion along the inclined slip plane rather than to dislocation emission.

The results in Table 4 show that the critical loading $G_{\mathrm{d}}$ increases with $\phi$. This is easily understood from eqn. (39); the screw component gives an extra factor of $\left[1+(1-v) \tan ^{2} \phi\right]$ to the critical loading $G_{\mathrm{d}}$
The percentage reduction, from the effective shear stress intensity approximation to the result of numerical solution of the shear-only model, is from $7 \%$ to $26 \%$. The percentage reduction depends to a large 
extent on the angle $\theta$, and generally to a lesser extent on the angle $\phi$. The larger the angle $\phi$ the smaller the reduction, and the larger the Poisson ratio the larger the reduction. For any fixed $\phi$ we see from Table 4 that the percentage reduction in $G_{\mathrm{d}}$, from the effective stress intensity approximation to the shear-only model, increases with angle $\theta$.

From the shear-only to the tension-shear coupling model, the percentage reduction depends on the $q$ and $p$ parameters of the material and slip system, and for the same parameters, on the effective phase angle $\psi$. For $\mathrm{Fe}$, the percentage reduction is $8 \%$ to $20 \%$, for $\mathrm{Al}$ $7 \%$ to $22 \%$, for $\mathrm{Ni} 7 \%$ to $20 \%$ and for $\mathrm{Ni}_{3} \mathrm{Al} 6.4 \%$ to $20 \%$.

However, for the $\mathrm{Si}$ glide set, the tension-shear coupling is very strong, and the percentage reduction is $19 \%$ to $40 \%$, neglecting the cases marked $\mathrm{C}$, to be discussed. For the $\mathrm{Si}$ shuffle set, surprisingly, the tension-shear coupling model gives a higher $G_{\mathrm{d}}$ than the shear-only model does because of the negative $p$ parameter in the one case not marked $\mathrm{C}$.

The estimate using the tension-reduced $\gamma_{\mathrm{us}}(\psi)$ of eqn. (31) in the shear only model works surprisingly well for $\mathrm{Fe}, \mathrm{Ni}, \mathrm{Al}$ and $\mathrm{Ni}_{3} \mathrm{Al}$, and gives an error less than $9 \%$. This method for the Si glide set gives an error less than $19 \%$, and for the shuffle set gives an error less than $11 \%$, excepting cases marked $\mathrm{C}$.

For $\mathrm{Si}$, both glide and shuffle sets, the tension-shear coupling model, because of strong coupling and the pure mode I loading, in a few cases renders solutions that have dominantly a decohesion profile and may possibly be better interpreted as a crack cleavage branching instability, rather than as a shear instability of dislocation nucleation. Such solutions are labeled C in Table 4.

The last column in Table 4 gives the percentage reduction from the effective stress intensity model to the tension-shear coupling model, which is $24 \%$ to $53 \%$.

\section{Summary}

The softening effect of the tensile stress across a slip plane, emanating from a crack tip, on dislocation nucleation from the crack tip has been investigated in a Peierls framework. The distributions of sliding and opening displacement along the slip plane, non-linearly coupled to the local shear and tensile stresses through a constitutive law based on atomistic calculations, are fully solved by an exact numerical method under tension and shear loadings, up to the critical condition for nucleating a full or partial dislocation line. By studying two possible cases where the normal stress is present, the case of the mixed tension and in-plane shear mode in the case of coplanar slip and crack planes $(\theta=0)$, and the case of pure tension loading but inclined slip plane, we have quantified the tension-shear coupling effects. Results are given for parameters based on EAM models of $\mathrm{Fe}, \mathrm{Al}, \mathrm{Ni}$ and $\mathrm{Ni}_{3} \mathrm{Al}$, and on a DFT-LDA model of Si. The general dependence on the tension-shear parameters $(q, p)$ over a wide range for $\theta=0$ and $\phi=0$ has been determined here.

We also found that the tension softening effect can be reasonably described by the tension-softened unstable stacking energy $\gamma_{\mathrm{us}}(\psi)=\gamma_{\mathrm{us}}{ }^{(\mathrm{r})}-\alpha\left[\gamma_{\mathrm{us}}{ }^{(\mathrm{u})}-\gamma_{\mathrm{us}}{ }^{(\mathrm{r})}\right]$ $(\pi / 2-\psi)$ which is utilized in a much simpler shear-only type of analysis. Here $\tan \psi$ is the ratio of the effective shear to tensile intensity factors along the slip plane (i.e. $\tan \psi=K_{\tau}^{\text {eff }} / K_{\sigma}^{\text {eff }}$, when $\theta=0$ and $\phi=0, \tan \psi=$ $\left.K_{\mathrm{II}} / K_{\mathrm{I}}\right)$. The tension softening coefficient $\alpha$ is near unity for all the metals studied, but is much larger for the $\mathrm{Si}$ glide set and is negative for the Si shuffle set. Another simple and approximate approach is to use the modified unstable stacking energy $\gamma_{\mathrm{us}}{ }^{\left(\mathrm{u}^{*}\right)}$ in the shear-only model although this does a poor job in the Si shuffle case.

We conclude that the tension eases dislocation nucleation at a crack tip, to an extent which can be described quantitatively by the tension-softened unstable stacking energy. In this work, we also treated nucleation of dislocations of mixed edge and screw components on an inclined slip plane. All our results correspond to loadings for spontaneous nucleation; thermal activation will allow nucleation at a finite rate and lower load levels.

\section{Acknowledgments}

The studies reported here have been supported by the Office of Naval Research, Mechanics Division (grants N00014-90-J-1379 and N00014-92-J-1960), and earlier phases of the work were supported by the National Science Foundation Materials Research Laboratory at Harvard (grant DMR-89-20490). Some of the computations were carried out under NSF support at the Pittsburgh Supercomputing Center. We are grateful for discussions with E. Kaxiras on DFT-LDA studies of stacking fault energies for $\mathrm{Si}$, with J. C. H. Spence and Y. M. Huang on Si $\{111\}$ surface energies, with S. M. Foiles on the (111) APB energy in $\mathrm{Ni}_{3} \mathrm{Al}$ as modeled in EAM, and with Y. L. Cui on the numerical procedure for solving the integral equations. In addition, we thank K. S. Cheung for providing us with EAM functions for Fe, R. G. Hoagland for Al, and $\mathrm{S}$. M. Foiles for $\mathrm{Ni}$ and $\mathrm{Ni}_{3} \mathrm{Al}$. Additionally, we are grateful to I.-H. Lin for supplying explicit algebraic representations of the dislocation stress functions $g_{i j}(r, s ; \theta)$. 


\section{References}

1 J. R. Rice, J. Mech. Phys. Solids, 40 (1992) 239.

2 R. E. Peierls, Proc. Phys. Soc., 52 (1940) 34.

3 G. Schoeck, Philos. Mag. A, 63 (1991) 111.

4 J. R. Rice and R. M. Thomson, Philos. Mag., 29 (1974) 73.

5 Y. Sun, J. R. Rice and L. Truskinovsky, in L. A. Johnson, D. T. Pope and J. O. Stiegler (eds.), High-Temperature Ordered Intermetallic Alloys, Materials Research Society, Pittsburgh, PA, 1991, Vol. 213, pp. 243-248.

6 S. M. Foiles, M. I. Baskes and M. S. Daw, Phys. Rev, B, 33 (1986) 7983.

7 S. M. Foiles and M. S. Daw, J. Mater. Res., 2 (1987) 5.

8 G. E. Beltz and J. R. Rice, in T. C. Lowe, A. D. Rollett, P. S. Follansbee and G. S. Daehn (eds.), Modeling the Deformation of Crystalline Solids, TMS, Warrendale, PA, 1991, p. 457.

9 G. E. Beltz and J. R. Rice, Acta Metall., 40 (Suppl.) (1992) s321.

10 J. R. Rice, Z. Suo and J.-S. Wang, in M. Ruhle, M. F. Ashby, A. G. Evans and J. P. Hirth (eds.), Metal-Ceramic Interfaces, Acta-Scripta Metall. Proc. Ser., Vol. 4, Pergamon, 1990, pp. 269-294.

11 G. E. Beltz and J.-S. Wang, Acta Metall, 40 (1992) 1675.

12 J. R. Rice, G. E. Beltz and Y. Sun, in A. S. Argon (ed.), Topics in Fracture and Fatigue, Springer, 1992, p. 1.

13 Y. Sun and G. E. Beltz, Anisotropic elastic formulation of dislocation nucleation, 1993, in preparation.

14 G. E. Beltz and J. R. Rice, The activation energy for dislocation nucleation at a crack tip, 1993, in preparation.

$15 \mathrm{~K}$. Cheung, PhD Thesis, Department of Nuclear Engineering, MIT, Cambridge, MA, 1990.

16 K. Cheung, A. S. Argon and S. Yip, J. Appl. Phys., 69(1991) 2088.

17 A. Kelly, W. R. Tyson and A. H. Cottrell, Philos. Mag., 15 (1967) 567.

18 A. S. Argon, Acta Metall., 35 (1987) 185.

19 I.-H. Lin and R. Thomson, Acta Metall., 34 (1986) 187.

20 G. E. Beltz, PhD Thesis, Division of Applied Sciences, Harvard University, Cambridge, MA, 1992.
21 F. Erdogan, in A. C. Eringen (ed.), Continuum Physics, Academic Press, 1975, p. 523.

22 F. Erdogan and G. D. Gupta, Q. Appl. Math., 29(1972) 525.

23 J. R. Rice, J. Appl. Mech., 35 (1968) 379.

24 J. R. Rice, in H. Liebowitz (ed.), Fracture: An Advanced Treatise, Vol. 2, Mathematical Fundamentals, Academic Press, New York, 1968, p. 191.

25 J. D. Eshelby, in M. F. Kanninen, W. F. Adler, A. R. Rosenfield and R. I. Jaffe (eds.), Inelastic Behavior of Solids, McGraw-Hill, New York, 1970, p. 77.

26 J. R. Willis, J. Mech. Phys. Solids, 15 (1967) 151.

27 J. Frenkel, Z. Phys., 37 (1926) 572

28 J. H. Rose, J. Ferrante and J. R. Smith, Phys. Rev. L., 47 (1981) 675

29 A. Needleman, J. Mech. Phys. Solids, $38(1990) 289$.

30 R. J. Harrison, F. Spaepen, A. F. Voter and A. F. Chen, in G. B. Olson, M. Azrin and E. S. Wright (eds.), Innovations in Ultrahigh-Strength Steel Technology, Plenum, 1990, p. 651

31 R. G. Hoagland, M. S. Daw, S. M. Foiles and M. I. Baskes, J. Mater. Res., 5 (1990) 313.

32 J. P. Hirth and J. Lothe, Theory of Dislocations, McGrawHill, New York, 1982, p. 839, 2nd edn.

33 W. H. Press, B. P. Flannery, S. A. Teukolsky and W. T. Vetterling, Numerical Recipes: The Art of Scientific Computing, Cambridge University Press, 1986, p. 521.

34 J. H. Rose, J. R. Smith, F. Guinea and J. Ferrante, Phys. Rev. $B, 29$ (1984) 2963.

35 S. M. Foiles, private communication, December 15, 1992.

36 E. Kaxiras and M. S. Duesbery, Phys. Rev. Lett., 70 (1993) 3752.

37 M. S. Duesbery, D. J. Michel, E. Kaxiras and B. Joos, in P. D. Bristowe, J. E. Epperson, J. E. Griffith and Z. LilientalWeber (eds.), Defects in Materials, Materials Research Society, Pittsburgh, PA, 1990, Vol. 209, pp. 125-130.

38 Y. M. Huang, J. C. H. Spence, O. T. Sankey and G. B. Adams, Surf. Sci., 256 (1991) 344-353.

39 Y. M. Huang, private communication, June 1992. 